\title{
In Vivo Rapid Investigation of CRISPR-Based Base Editing Components in Escherichia coli (IRI-CCE): A Platform for Evaluating Base Editing Tools and Their Components
}

\author{
Rahul Mahadev Shelake ${ }^{1, *,+} \mathbb{D}$, Dibyajyoti Pramanik ${ }^{1,+} \mathbb{D}$ and Jae-Yean Kim ${ }^{1,2, *}$ \\ 1 Division of Applied Life Science (BK21 FOUR Program), Plant Molecular Biology and Biotechnology Research \\ Center, Gyeongsang National University, Jinju 52828, Korea; dpinbiotech@gmail.com \\ 2 Division of Life Science, Gyeongsang National University, 501 Jinju-daero, Jinju 52828, Korea \\ * Correspondence: rahultnau@gmail.com (R.M.S.); kimjy@gnu.ac.kr (J.-Y.K.) \\ + These authors contributed equally as co-first authors to this work.
}

check for updates

Citation: Shelake, R.M.; Pramanik,

D.; Kim, J.-Y. In Vivo Rapid Investigation of CRISPR-Based Base Editing Components in Escherichia coli (IRI-CCE): A Platform for Evaluating Base Editing Tools and Their Components. Int. J. Mol. Sci. 2022, 23, 1145. https://doi.org/ 10.3390/ijms 23031145

Academic Editor: Giorgio Dieci

Received: 10 December 2021

Accepted: 18 January 2022

Published: 20 January 2022

Publisher's Note: MDPI stays neutral with regard to jurisdictional claims in published maps and institutional affiliations.

Copyright: (C) 2022 by the authors. Licensee MDPI, Basel, Switzerland. This article is an open access article distributed under the terms and conditions of the Creative Commons Attribution (CC BY) license (https:// creativecommons.org/licenses/by/ $4.0 /)$.

\begin{abstract}
Rapid assessment of clustered regularly interspaced short palindromic repeats/CRISPRassociated protein (CRISPR/Cas)-based genome editing (GE) tools and their components is a critical aspect for successful GE applications in different organisms. In many bacteria, double-strand breaks (DSBs) generated by CRISPR/Cas tool generally cause cell death due to the lack of an efficient nonhomologous end-joining pathway and restricts its use. CRISPR-based DSB-free base editors (BEs) have been applied for precise nucleotide (nt) editing in bacteria, which does not need to make DSBs. However, optimization of newer BE tools in bacteria is challenging owing to the toxic effects of $\mathrm{BE}$ reagents expressed using strong promoters. Improved variants of two main BEs, cytidine base editor (CBE) and adenine base editor (ABE), capable of converting $C$ to $T$ and $A$ to $G$, respectively, have been recently developed but yet to be tested for editing characteristics in bacteria. Here, we report a platform for in vivo rapid investigation of CRISPR-BE components in Escherichia coli (IRI-CCE) comprising a combination of promoters and terminators enabling the expression of nCas9-based $\mathrm{BE}$ and sgRNA to nontoxic levels, eventually leading to successful base editing. We demonstrate the use of IRI-CCE to characterize different variants of CBEs (PmCDA1, evoCDA1, APOBEC3A) and ABEs (ABE8e, ABE9e) for bacteria, exhibiting that each independent BE has its specific editing pattern for a given target site depending on protospacer length. In summary, CRISPR-BE components expressed without lethal effects on cell survival in the IRI-CCE allow an analysis of various BE tools, including cloned biopart modules and sgRNAs.
\end{abstract}

Keywords: base editing; CRISPR; genome editing; heterologous expression

\section{Introduction}

The adoption of the bacterial immune system, clustered regularly interspaced short palindromic repeats/CRISPR-associated protein (CRISPR/Cas), for targeted genetic manipulation has transformed the field of genome editing (GE) research [1]. The CRISPR-based tools allow direct or indirect programmable engineering of all three major building blocks of life, i.e., DNA, RNA, and protein [2]. In eukaryotes, double-strand breaks (DSBs) generated by CRISPR/Cas tool are repaired by error-prone nonhomologous end-joining (NHEJ) pathway leading to the gene disruption. Most bacteria lack the efficient NHEJ pathway, thus restricting the DSB-dependent CRISPR/Cas applications [3]. The DSB-free DNA base editors (BEs) are emerging tools to install precise mutations at target regions in bacteria [4].

In BE systems, deaminase fusion with Cas enzyme allows precise base conversion in the targeted region. The BE tools are based on the use of nickase (nCas9) or dead Cas9 (dCas9) (mainly from Streptococcus pyogenes) that does not need to make DSBs in the target DNA $[5,6]$. In animals and plants, primarily nCas9-BE is employed to stimulate the nicking of non-deaminated strands that produces single-strand breaks (SSBs) and favor desired 
BE outcomes by tweaking the cellular DNA damage responses [7]. The single guide RNA (sgRNA) together with the nCas9-BE or dCas9-BE complex binds the protospacer adjacent motif (PAM) in the target locus to form an R-loop, in which the partially open nontarget strand (NTS) provides a possible substrate for base conversion. Depending on the type of deaminase used, editing can occur within or near the protospacer, described as deamination or editing window [8].

Base editing tools have recently been optimized in various microbes due to their simplicity, high efficiency, and precision. However, toxic effects of BE components (deaminase, nCas9(D10A), uracil-DNA glycosylase inhibitor (UGI) protein) were earlier reported in several bacterial species (Table 1). For example, expression of some components of CRISPR-BE machinery shown poor transformation efficiency, such as nCas9(D10A), nCas9(D10A)PmCDA, and dCas9-PmCDA1-1 $\times$ UGI in Escherichia coli [9]; rAPOBEC1-nCas9(D10A) in Pseudomonas sp. [10]; nCas9(D10A)-PmCDA1 in Bacillus subtilis [11,12]; and dCas9PmCDA1-1 ×UGI-LVA.Tag in Streptomyces spp. [13] and Agrobacterium sp. [14]. Higher expression of BE reagents was reported to adversely affect cell growth and BE efficiency [12]. The use of an optimal promoter facilitated successful base editing in Bacillus sp. [12], indicating an appropriate amount of BE expression as a crucial factor in achieving higher BE efficiency. Nevertheless, using an inducible promoter [10] or optimal constitutive promoter [15] circumvents the toxic effects of BE components. Previously, inducible promotermediated leaky expression (even without adding the inducer chemicals) was sufficient to achieve efficient base editing [11-14,16]. In this regard, we propose finding the promoters expressing the optimal amount of BE components, including sgRNAs that may help avoid cell toxicity, and analyzing BE tools. The use of validated CRISPR-BE biopart modules in Golden Gate assembly protocols [17] might be helpful for both prokaryotic and plant BE experiments.

Here, a BE system was employed to establish an efficient and rapid evaluation platform to assess the differential-strength promoter-driven base conversion in E. coli, termed in vivo rapid investigation of CRISPR-BE components in E. coli (IRI-CCE). We report for the first time that the efficient sgRNA expression was driven by the consensus sequence of Arabidopsis U6 promoter (pAtU6) (75 bp) in E. coli cells. The modular cloning approach described in the present work enables the validation of designed bioparts for further modular cloning of CRISPR-BE plasmids and the interchangeable use of bioparts independent of its context in the IRI-CCE platform. Different versions of cytidine base editor (CBE) and adenine base editor (ABE) were analyzed for editing features and substitution efficiency from cytosine to thymine ( $C$ to $T$ ) and adenine to guanine (A to $G$ ), respectively. Editing characteristics of BEs, including CBEs (Target-AID system based on sea lamprey cytidine deaminase, PmCDA1 [6]; evolved variant of PmCDA1, evoCDA1 [18]; human APOBEC3A, A3A [19] and ABEs (ABE8e [20] and ABE9e [21]) were optimized. This is the first report of optimization of evoCDA1, APOBEC3A, ABE8e, ABE9e, and pAtU6-mediated gRNA expression for BE studies in E. coli. Moreover, editing outcomes of the analyzed set of spacers (gRNAs) of different lengths imply that the gRNA length is crucial in achieving variable sizes of editing windows. 
Table 1. Toxicity of base editor (BE) components reported in bacterial base editing studies. Editing window numbers counted from the distal of PAM in 20 bp spacers. Promoter-BE reagent combinations exhibiting toxic effects on cell survival are highlighted in gray.

\begin{tabular}{|c|c|c|c|c|c|c|c|c|}
\hline Species & gRNA Promoter & BE Type & Cas9 & BE Promoter & Construct Scheme & $\begin{array}{l}\text { Editing } \\
\text { Window }\end{array}$ & $\begin{array}{c}\text { Cell } \\
\text { Toxicity }\end{array}$ & References \\
\hline \multirow{3}{*}{ Escherichia coli } & J23119 (Const.) & PmCDA1 & nCas9 & Lambda (Induc.) & $\begin{array}{l}\text { Lambda-nCas9-3xFLAG-SH3Link- } \\
\text { PmCDA1-1×UGI }\end{array}$ & - & Toxic & [9] \\
\hline & J23119 (Const.) & PmCDA1 & dCas9 & Lambda (Induc.) & $\begin{array}{c}\text { Lambda-dCas9-SH3Link-3xFLAG- } \\
\text { PmCDA1-1×UGI }\end{array}$ & - & Toxic & \\
\hline & J23119 (Const.) & PmCDA1 & dCas9 & Lambda (Induc.) & $\begin{array}{c}\text { Lambda-dCas9-3xFLAG-PmCDA1- } \\
1 \times \text { UGI-LVA.Tag }\end{array}$ & 1 to $5 \#$ & Nontoxic & \\
\hline \multirow{7}{*}{$\begin{array}{l}\text { Pseudomonas putida } \\
\text { KT2440 }\end{array}$} & J23119 (Const.) & rAPOBEC1 & nCas9 & Pbs (Const.) & Pbs-rAPOBEC1-nCas9 & - & Toxic & [10] \\
\hline & J23119 (Const.) & rAPOBEC1 & enCas9 * & Pbs (Const.) & Pbs-rAPOBEC1-enCas9 & - & Toxic & \\
\hline & J23119 (Const.) & rAPOBEC1 & nCas9 & ParaBAD (Induc.) & ParaBAD-rAPOBEC1-nCas9 & - & Toxic & \\
\hline & J23119 (Const.) & rAPOBEC1 & enCas9 & ParaBAD (Induc.) & ParaBAD-rAPOBEC1-enCas9-1×UGI & 3 to 8 & Nontoxic & \\
\hline & J23119 (Const.) & rAPOBEC1 & enCas9-NG & ParaBAD (Induc.) & ParaBAD-rAPOBEC1-enCas9-NG-1×UGI & 3 to 8 & Nontoxic & \\
\hline & J23119 (Const.) & rAPOBEC1 & enCas9 & $P_{\text {Xyls }}$ (Induc.) & Xyls-rAPOBEC1-enCas9-1×UGI & ND & Nontoxic & \\
\hline & J23119 (Const.) & $\begin{array}{l}\text { rAPOBEC1 (W90Y, } \\
\text { R126E) }\end{array}$ & enCas9 & ParaBAD (Induc.) & $\begin{array}{c}\text { ParaBAD-rAPOBEC1(W90Y, } \\
\text { R126E)-enCas9-1×UGI }\end{array}$ & 4 to 7 & Nontoxic & \\
\hline \multirow{3}{*}{ Bacillus subtilis } & $\mathrm{P}_{\text {veg }}$ (Const.) & - & nCas9 & $P_{\text {grac }}$ (Induc.) $* *$ & $\mathrm{P}_{\text {grac }}-\mathrm{nCas} 9$ & - & Toxic & [11] \\
\hline & $P_{\text {veg }}$ (Const.) & PmCDA1 & nCas9 & $\mathrm{P}_{\text {grac }}($ Induc.) $* *$ & $P_{\text {grac }}$-nCas9-PmCDA1 & - & Toxic & \\
\hline & $\mathrm{P}_{\mathrm{veg}}$ (Const.) & PmCDA1 & dCas9 & $P_{\text {grac }}\left(\right.$ Induc.) ${ }^{* *}$ & $\mathrm{P}_{\text {grac }}$-dCas9-PmCDA1 & 1 to 5 & Nontoxic & \\
\hline \multirow{3}{*}{ Bacillus subtilis } & $\operatorname{araABCD}$ (Induc.) & - & nCas9 & $P_{\text {grac }}($ Induc.) $* *$ & $\mathrm{P}_{\text {grac }}-\mathrm{nCas} 9$ & - & Toxic & [12] \\
\hline & $\operatorname{araABCD}$ (Induc.) & PmCDA1 & dCas9 & Pgrac (Induc.) $* *$ & $P_{\text {grac }}$-dCas9-PmCDA1-1×UGI-LVA.Tag & 1 to 5 & Nontoxic & \\
\hline & ara $\mathrm{ABCD}$ (Induc.) & rAPOBEC1 & dCas9 & $\mathrm{P}_{\text {grac }}($ Induc.) $* *$ & $\begin{array}{c}\text { Prac }_{\text {grAPOBEC1-dCas9-PmCDA1- }} \\
1 \times \text { UGI-LVA.Tag }\end{array}$ & ND & Nontoxic & \\
\hline $\begin{array}{l}\text { Paenibacillus } \\
\text { polymyxa } \mathrm{E} 681\end{array}$ & ara $\mathrm{ABCD}$ (Induc.) & PmCDA1 & dCas9 & $\mathrm{P}_{\text {spac }}\left(\right.$ Induc.) ${ }^{* *}$ & $P_{\text {spac-dCas9-PmCDA1-1×UGI-LVA.Tag }}$ & ND & Nontoxic & \\
\hline \multirow[b]{2}{*}{ Agrobacterium spp. } & J23119 (Const.) & PmCDA1 & dCas9 & PaadA (Const.) & PaadA-dCas9-PmCDA1-1×UGI-LVA.Tag & & Toxic & [14] \\
\hline & J23119 (Const.) & PmCDA1 & dCas9 & PvirB (Induc.) ${ }^{* *}$ & PvirB-dCas9-PmCDA1-1×UGI-LVA.Tag & ND & Nontoxic & \\
\hline
\end{tabular}

* enCas9, enhanced Cas9 (K848A/K1003A/R1060A); ** leaky expression by inducible promoter was sufficient for efficient base editing; Const., constitutive promoter; Induc., inducible promoter; UGI, uracil DNA glycosylase inhibitor; ND, no data or analysis done about editing window; \# editing window varies with gRNA length. 


\section{Result}

2.1. Optimizing Base Editing Platform Based on PmCDA1-Mediated C-to-T Conversion in E. coli

The CRISPR-based BE construct consists of two major transcriptional units: deaminasefused Cas9 mutant and sgRNA expression unit, driven by two separate promoters. Previous bacterial BE studies consisted of a deaminase fusion with partially impaired nCas9 or inactive dCas9 $[9,14,22,23]$. We chose the nCas9(D10A)-based CBE system in the present study because of the frequent use in BE studies in different organisms. Partially active nCas9 generates the SSBs that favor desired BE outcomes [7].

\subsubsection{Selecting Nontoxic Promoters for BE Studies in E. Coli}

As observed in earlier studies, the higher expression of BE components adversely affects cell survival in bacteria $[9,14]$. To avoid the lethal effect of nCas9-BE (examples of cell toxicity also summarized in Table 1), we opted to explore different promoters for the expression of CRISPR-BE machinery without affecting cell survival. Among the tested promoters, we observed poor transformation efficiency with a mix of incorrect clones in the case of high-strength bacterial promoter pEc1 for CBE testing (Figures S1 and S2). When CBE (nCas9(D10A)-PmCDA1-1×UGI or dCas9(D10A+H840A)-PmCDA1-1UGI) was expressed by $\mathrm{pEc1}$ together with pJ23119-sgRNA, no correct clones were obtained, possibly due to the toxicity caused by BE components in E. coli (Table S1).

Bacterial pGlpT (BBa_J72163) promoter [24,25] showed optimal expression activity but less activity than pEc1 in transcript analysis of superfolder green fluorescent protein (sfGFP) (Figure S2). Additionally, cauliflower mosaic virus (CaMV) 35S promoter (p35S) was selected to evaluate the expression activity of nCas9-BE components in E. coli. Although the p35S is less active in E. coli [26,27], this aspect is of great significance for rapidly validating cloned plasmids in E. coli or designing a heterologous expression platform consisting of differential-strength promoters (Figure 1A). The use of validated CRISPR-BE biopart modules in Golden Gate assembly protocols might be helpful for BE experiments allowing compatible use in both bacteria and plants. In several instances, cloning new plasmid vectors, genetic delivery of CRISPR plasmids, and analysis of GE outcomes are costly and time-consuming processes [28,29]. Thus, a platform for validating cloned BE plasmids, including sgRNA components, may save the investment of resources and time. Incidentally, plasmid vectors with the ability of ectopic expression of Cas enzyme and sgRNAs in bacteria will facilitate the quick screening and reliability of cloned plasmids and editing activities of designed gRNAs. Among the analyzed p35S versions (p35S long with or without intron and p35S short), intron-containing p35S labeled as p35S(L)I [17] showed moderate sfGFP expression activity (data not shown). Therefore, two differential-strength promoters, high-strength pGlpT and low-strength p35S(L)I, were selected for nCas9-BE expression in E. coli.

We sought to inspect 10 different RNA polymerase III (RNA Pol III)-dependent promoters for conserved -10 (TATAAT) and -35 (TTGACA) elements (Figure S3). The promoters were chosen because of earlier studies reporting efficient sgRNA expression in different species, including bacteria (pJ23119), plants (pAtU6, pOsU3, pOsU6, pMtU6.6, pZmU3, pTaU3, pTaU6), yeast (pSNR52), and human (phU6). The pJ23119 promoter has successfully been used for sgRNA expression in $E$. coli and other bacteria $[9,14,30]$. We detected partial or fully conserved -10 and -35 elements in all the promoters. Remarkably, the DNA sequence alignment of pJ23119 and pAtU6 showed 54.28\% identity with fully conserved -10 and partially conserved -35 elements (Figure 1B). Therefore, in view of developing a heterologous promoter for sgRNA expression, pAtU6 was examined for optimal sgRNA expression in E. coli. 
A

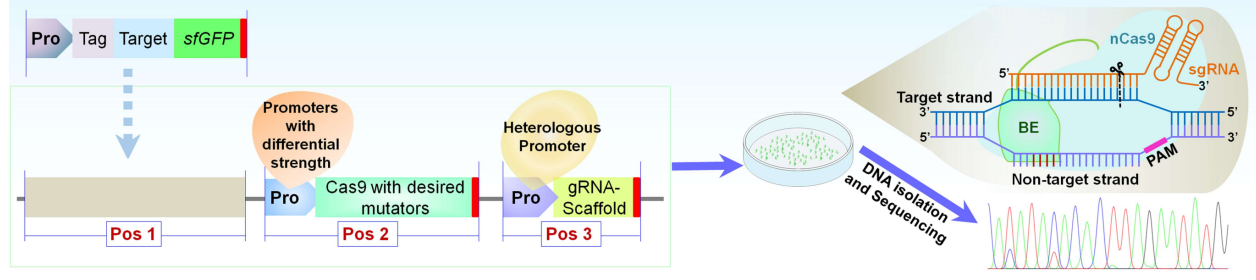

B

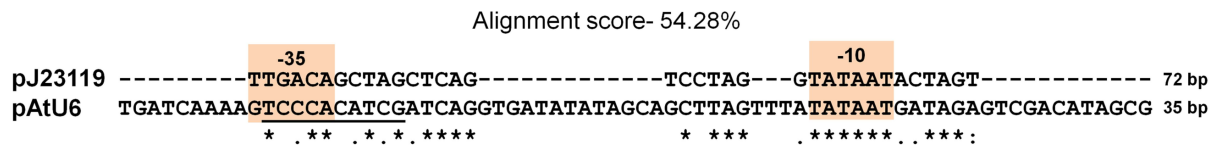

C

Two-plasmid based assay

Component 1: Target Module (Carbenicillin selection)

\begin{tabular}{|l|l|l|l|l|}
\hline Pro & Tag & Target site & sfGFP & Ter \\
\hline
\end{tabular}

Component 2: BE and sgRNA Expression Modules

(Kanamycin selection)

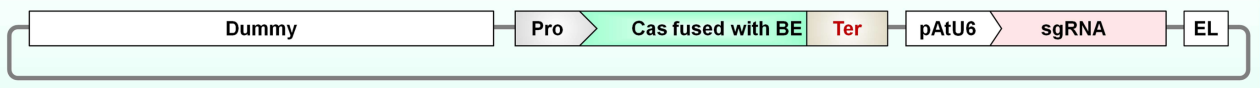

D

Single-plasmid based assay

(Kanamycin selection)

\begin{tabular}{|l|l|l|l|l|l|l|l|l|l|l|l|l|l|l|l|l|l|}
\hline Pro & Pag & Target site & sfGFP & Ter & Sas fused with BE & Ter \\
\hline
\end{tabular}

Figure 1. Schemes used for optimization of in vivo rapid investigation of CRISPR-BE components in Escherichia coli (IRI-CCE) platform. (A) Schematic representation of IRI-CCE platform. Promoters of differential strength may enable the production of CRISPR-BE components (Cas9-fusions and sgRNAs) without toxic effects in E. coli, allowing the testing of new BE tools, reliability of cloned plasmids, and editing activities of designed sgRNAs. (B) Sequence alignment of bacterial pJ23119 and Arabidopsis U6 (AtU6) promoter. The features of AtU6 promoter include two conserved elements: the upstream sequence element (USE; consensus sequence RTCCCACATCG) and a TATA-like box (consensus sequence TTTATATA) which are highlighted using underline and black box, respectively. "** denote identical nucleotides, ":" denote conserved nucleotides, and "." symbol denote semiconserved nucleotides. (C) Two approaches analyzed for the establishment of base-editing-based IRI-CCE platform. Two-plasmid-based assay comprises two independent plasmids. The first plasmid consisted of the target-region-expression plasmid with C-terminal superfolder green fluorescent protein (sfGFP). The second plasmid consists of an assemblage of promoter-sgRNA and promoternCas9-PmCDA1-linker-1×UGI-terminator (nCas9-PmCDA1) in a plasmid vector. (D) A singleplasmid assay system composed of all three parts assembled into a single plasmid, including target region, promoter-sgRNA, and nCas9-PmCDA1.

\subsubsection{Modular Design for Desired Target DNA Cloning and Platform Optimization}

We employed a PmCDA1-based Target-AID system that induces C-to-T mutations in the editing window located approximately 1 to 8 bases from the distal end of the PAM (counting positions 21-23 for PAM) in $20 \mathrm{bp}$ protospacer [6]. We adopted two approaches for BE testing. Firstly, a two-plasmid system consisted of a target plasmid and a CRISPR plasmid including Pro-sgRNA and Pro-nCas9(D10A)-PmCDA1-1×UGI (Figure 1C). Secondly, a single-plasmid system was composed of the target, sgRNA, and PronCas9(D10A)-PmCDA1-1×UGI assembled into a single vector (Figure 1D). We analyzed 
both single- and two-plasmid vector systems, showing similar editing outcomes (Figure S4). Therefore, a single-plasmid system was used for further analysis.

Next, the target regions for the Test sgRNAs were synthesized and cloned into the designed universal target-acceptor module (Figure 2). The intended $23 \mathrm{bp}$ target sequence (20 bp guide +3 bp PAM) was cloned using BsmBI type IIS sites in such a manner so that codons would be in frame with the sfGFP sequence. The sfGFP sequence facilitates the screening of successfully cloned plasmids depending on the presence or absence of fluorescence. Cloning of the required target DNA region into an sfGFP-based universal target-acceptor using MoClo kit protocol [17] permits the assessment of any intended sgRNA.
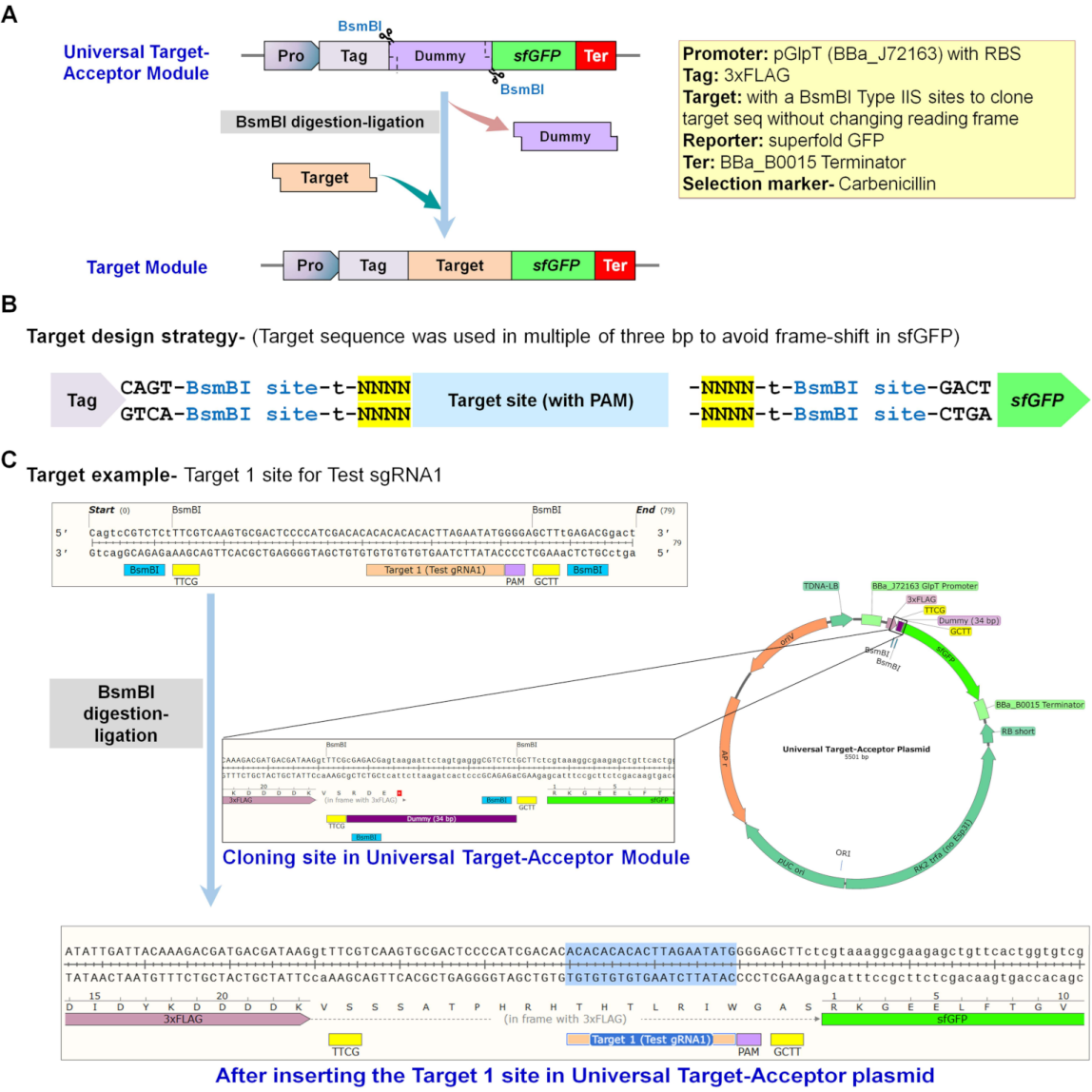

Figure 2. Scheme followed for cloning the target regions as DNA templates for sgRNA binding and subsequently CRISPR-BE editing. (A) Schematic representation of cloning region in the universal target-acceptor module. Different components used for assembly are summarized in the yellow box. Cloning of the desired target region into a universal target-acceptor through type IIS (BsmBI) enzyme digestion-ligation permits the assessment of any intended sgRNA. (B) The intended $23 \mathrm{bp}$ target sequence (20 bp guide $+3 \mathrm{bp}$ PAM) was cloned using BsmBI type IIS sites in such a manner so that codons would be in frame with the sfGFP sequence. The sfGFP sequence facilitates the screening of successfully cloned plasmids depending on the presence or absence of fluorescence. (C) Example of target cloning for Test sgRNA1 is shown.

Two sgRNAs were tested containing alternate $\mathrm{Cs}$ at even (test sgRNA1 target region, Figure $3 \mathrm{~A}$ ) and odd (test sgRNA2 target region, Figure $3 \mathrm{~B}$ ) positions spanning from 1 to 10 in the 20 bp protospacer. After transforming individual constructs in 10-beta E. coli, single colonies were cultured for plasmid isolation and target-region sequencing. Base editing frequency (ratio of edited colonies to total screened colonies containing at least one C-to-T in editable window) of nCas9-PmCDA1-1×UGI was found to be $100 \%$ in the case of pGlpT and p35S(L)I with variable efficiency in the editing window ranging 
from 1 to 6 position (Figure 3C,D). Calculated editing efficiency for different Cs within the editable window showed a variable percentage of C-to-T editing, implying that after transforming the plasmid vectors into competent cells, the grown individual clones were indeed mixed populations. Similar outcomes for base editing were previously reported in other bacteria, such as Corynebacterium glutamicum [31], Clostridium beijerinckii [32], Staphylococcus aureus [22], and Agrobacterium sp. [14]. For pGlpT, four colonies showed an average of $92 \%$ editing at targeted C4 in Test sgRNA1 target region and 79.5\% editing at targeted $\mathrm{C} 3$ in Test sgRNA2 target region (Figure 3C,D). A variable range of editing efficiencies was observed for each C position in nCas9(D10A)-PmCDA1-1 $\times$ UGI driven by different promoters, possibly due to differential expression of CRISPR-BE reagents. Moreover, base editing events with a single, clear genotype can be achieved from mixed population by streak plating (Figure 3E). Besides, the nCas9/dCas9-based pGlpT-CBE system together with pJ23119-sgRNA showed no correct clones (Table S1). The combination of nCas9/dCas9-based pGlpT-CBE and pAtU6-sgRNA permitted sufficient cell survival allowing successful $\mathrm{BE}$ analysis. These data indicate that the choice of promoters (driving $\mathrm{BE}$ or sgRNA) is a crucial parameter to avoid the lethal effects in BE experiments (Figure S5). Consequently, the optimized method including differential-strength promoters for expression of CRISPR components was named IRI-CCE, an in vivo rapid investigation of CRISPR components in E. coli. The IRI-CCE can be expanded by screening more nontoxic promoters with a wide range of strength in the future.

A

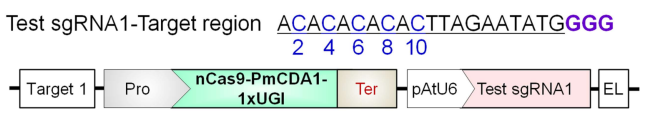

C

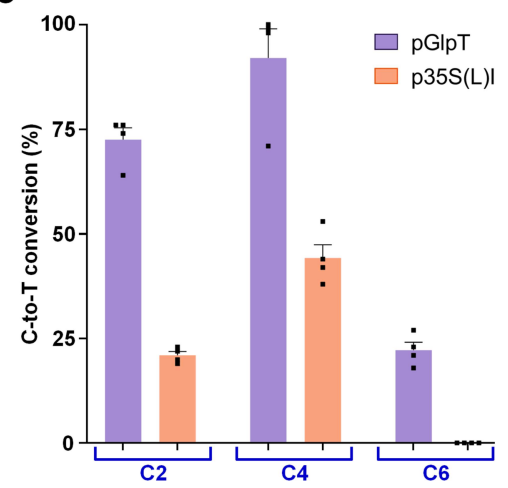

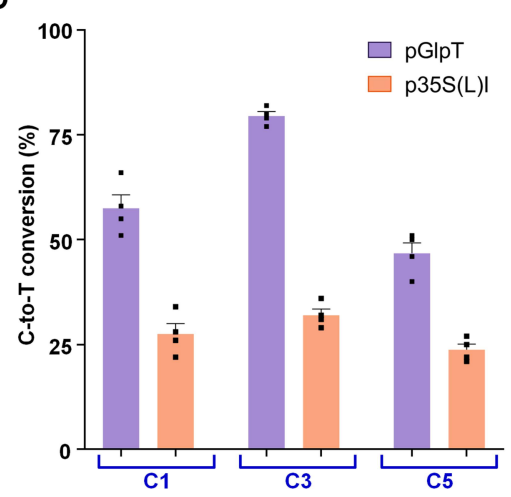

B

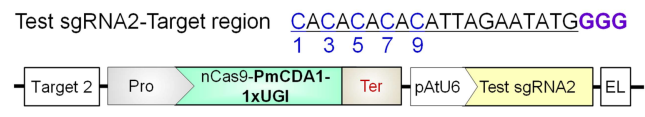

E

Figure 3. Evaluation of C-to-T conversion by PmCDA1-mediated cytosine base editor (CBE) driven by different promoters in E. coli cells. (A,B) Two independent sgRNAs were tested containing alternate Cs at even (Test sgRNA1, (A)) and odd (Test gRNA2, (B)) positions spanning from 1 to 10 in the $20 \mathrm{bp}$ protospacer length. The numbers are assigned by counting the distal base as 1 from the protospacer adjacent motif (PAM), i.e., GGG. Schematic representation of the plasmid vectors showing a synthesized target region for sgRNA and two transcriptional units (TUs) composed of nCas9 (D10A) fused with PmCDA1-1 $\times$ UGI and AtU6 promoter-sgRNA unit. (C,D) PmCDA1-based C-to-T editing activities. The base conversion rate was estimated using the online tool EditR. Graph values show the mean percentage on the $y$-axis and the tested protospacer positions on the $x$-axis. The graph bar shows the mean of percentage values, and error bars indicate the standard error of the mean (mean \pm s.e.m.) of four independent biological replicates. Dots indicate the individual biological replicates. (E) Sanger sequencing data of Test gRNA1 targeting region from the colony obtained directly after transformation (initial colony). Sanger sequencing data obtained after streak plating of initial clone culture (final clone). The substituted base is marked with a red inverted triangle. 


\subsection{Assessing Base Editing Activities on Chromosomal Targets in E. coli Genome}

Next, we targeted E. coli genes to evaluate the BE efficacy and potential toxicity mediated by differential-strength promoters in the genomic context of bacteria. Five different sgRNAs were chosen for editing three genes, namely galK, $r p o B$, and $r p p H$ (Figure 4). The 10-beta strain lacks the galK locus; therefore, $\mathrm{DH} 5 \alpha$ strain was used for examining the CBE-mediated editing of the galK locus. The pGlpT and p35S(L)I-driven nCas9(D10A)PmCDA1-1 $\times$ UGI efficiently edited the available Cs in the editing window of all the tested sites with sufficient cell survival for BE investigations.

A

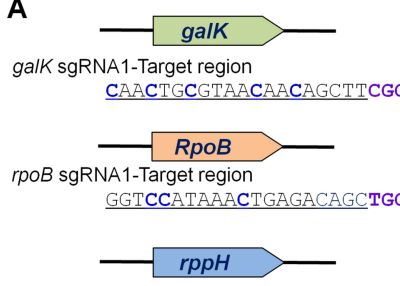

rppH sgRNA1-Target region ATCGCCAGGGGCAGGTAATGTGG

$r p p H$ sgRNA2-Target region AGGGGCAGGTAATGTGGGCCCGG

rppH sgRNA3-Target region TCCTGGCAATTTCCGCAAGGCGG

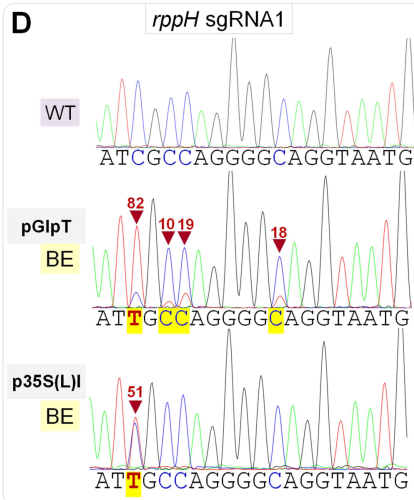

B

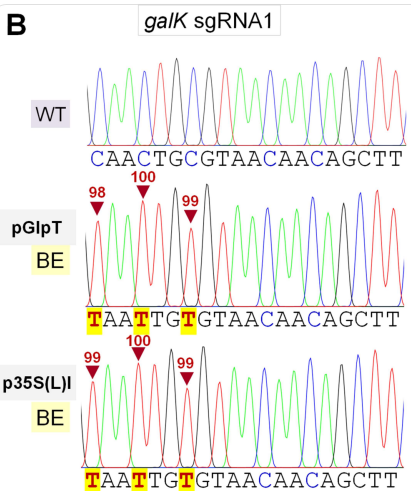

E

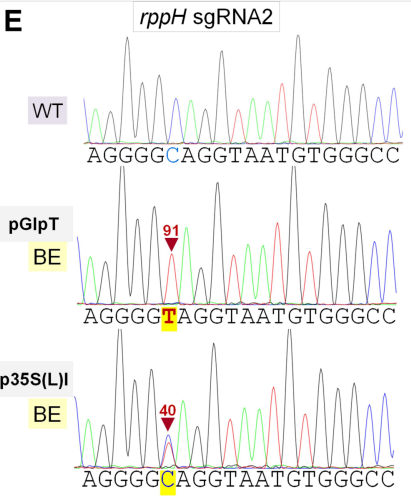

C $\quad$ rpoB sgRNA1

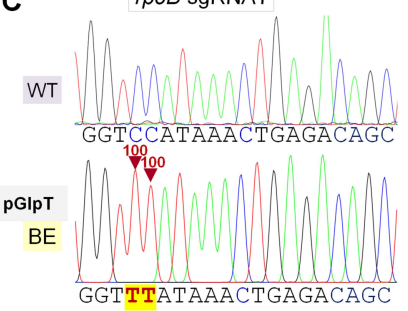

p35s(L)।

$\mathrm{BE}$

$\stackrel{94}{77}$

(1)

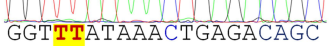

F $\quad r p p H$ sgRNA3

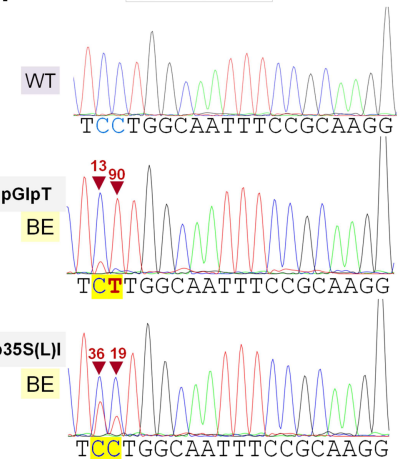

Figure 4. Differential-strength promoter-driven C-to-T editing activities by PmCDA1 in E. coli genome. (A) Designed sgRNAs and PAM site (violet) for targeting three genes in the $E$. coli genome. Out of five sgRNAs, 1, 1, and 3 were used for targeting galK, $r p o B$, and $r p p H$, respectively. All the cytosine nucleotides located in the protospacer are highlighted in blue (bold). (B-F) Sequence alignment of the PmCDA1-edited mutants. The pGlpT and p35S(L)I-driven Target-AID efficiently edited the available Cs in the editing window of all the tested sites. Notably, in the p35S(L)I-driven Target-AID system, higher C-to-T activity was observed in galK and rpoB, which might be attributable to the limited number of available $\mathrm{Cs}$ in the editing window compared to multicopy plasmid-encoded targets. The substituted base is marked with a red inverted triangle.

Because the editing efficiency of BE versions may differ due to distinct genetic backgrounds in different $E$. coli strains, we also examined the cytotoxicity and base conversion efficiency in three more strains, including DH5 $\alpha$, DB3.1, and BL21(DE3) for Test sgRNA1 target region using nCas9(D10A)-PmCDA1-1 $\times$ UGI. Irrespective of the genetic background of strain, we found sufficient cell survival allowing BE assays (Figure S6) that confirmed comparable C-to-T mutations in tested sgRNAs in all the four bacterial strains (Figure 5), suggesting the broader applicability of the IRI-CCE platform across different $E$. coli strains. 


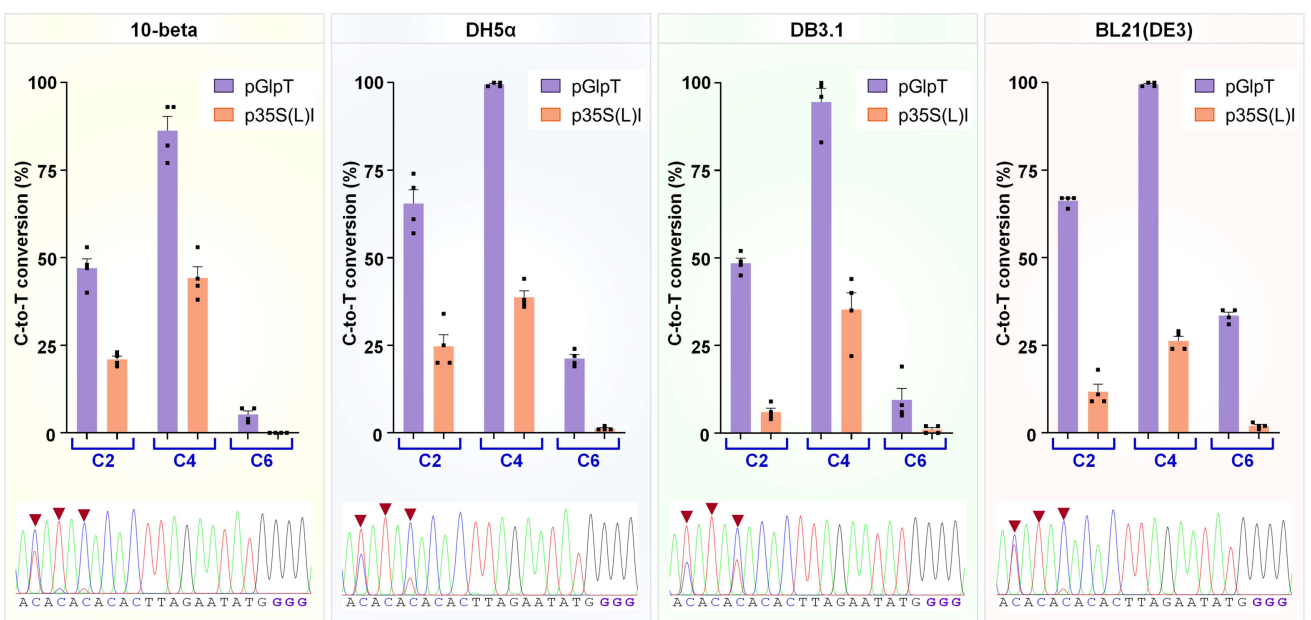

Figure 5. Evaluation of C-to-T conversion by PmCDA1-mediated cytosine base editor driven by pGlpT and p35S(L)I in E. coli strains: 10-beta, DH5 $\alpha$, DB3.1, and BL21(DE3). C-to-T editing activities at the synthetic target of Test gRNA1 were examined. Graph values show the mean percentage on the $y$-axis and the tested protospacer positions on the $x$-axis. The graph bar shows the mean of percentage values, and error bars indicate the standard error of the mean (mean \pm s.e.m.) of four independent biological replicates. Dots indicate the individual biological replicates. Representative example of Sanger sequencing data of Test gRNA1 targeting region including GGG as a protospacer adjacent motif (PAM) is shown on the bottom side of each panel. The substituted base is marked with a red inverted triangle.

\subsection{Optimization of Broad-Range CBEs Using IRI-CCE Platform}

Base editors with broader editing windows are generally applied for mutagenesis of a user-defined region. The editing windows vary according to BE type, target site, sequence context, and experimental conditions [7]. Several latest BE tools are not yet characterized for E. coli use. Mainly, features of evoCDA1, APOBEC3A, ABE8e, and ABE9e with enhanced BE activity in other organisms are not yet analyzed in bacteria. To characterize the editing features in E. coli, two CBE types (evoCDA1 and A3A) were tested in the IRI-CCE platform with distinctive features (different range of editing window and sequence context-preference). In original reports, editing windows for evoCDA1 and A3A were positioned from 1 to 14 [18] and 1 to 17 [19] with no sequence context-preference for evoCDA1 and moderate $\mathrm{CT}$ preference for $\mathrm{A} 3 \mathrm{~A}$. A core-editing region was observed between positions 3 to 12 for evoCDA1 in human cells and positions 3 to 9 for A3A in plants. To examine C-to-T conversion in E. coli, in addition to two Test sgRNAs, we designed Test sgRNA3 by mimicking the sequence of TaVRN1-sgRNA1 (Figure 6A) that comprises the Cs in the range of 1 to $17 \mathrm{bp} \mathrm{[19].}$

Constitutive expression of CRISPR components using different promoters showed an expanded editing window for evoCDA1 and A3A, possibly owing to higher nCas9-BE expression. Among the tested CBEs, evoCDA1 showed the C-to-T conversion with the broadened editing window from positions -6 to 14 with $\mathrm{pGlpT}$ and from positions -1 to 9 with p35S(L)I (Figure 6B). The expression of nCas9-A3A using pGlpT and p35S(L)I promoter also displayed distinct editing windows ranging from -6 to 17 and 2 to 17 , respectively (Figure 6C). For pGlpT-evoCDA1, C-to-T conversion at positions -1 to 9 was the highest (18-100\%), whereas for pGlpT-A3A, C-to-T mutation at positions 3 to 10 was the highest $(43-100 \%)$.

On the other hand, for p35S(L)I-evoCDA1, C-to-T conversion at positions 2 to 7 was the highest $(57-100 \%)$, whereas for p35S(L)I-A3A, C-to-T mutation at positions 4 to 10 was the highest (38-100\%). Notably, G:C at position -5 was mutated to A:T in both the CBE types with moderate efficiency across all the three sgRNAs (up to $43 \%$ ), implying the $\mathrm{C}$-to-T conversion in the target (non-deaminated) strand. In the case of CBEs, noncanonical $\mathrm{BE}$ outcomes at PAM-distal positions in the target region were also reported in human 
cells $[33,34]$. The upstream (positions -3 to -1 ) regions of target sites were not accessible to A3A deamination in the tested combinations of sgRNAs and respective target sites (Figure 6C). In addition, A3A-mediated C-to-T editing at middle positions (12 to 16) in Test sgRNA3 was not observed. The comparison between nCas9(D10A) and the CBE types (PmCDA1, evoCDA1, and A3A) containing rppH sgRNA3 targeting rppH gene indicated that all CBEs are less toxic with slight variation between pGlpT and p35S(L)I promoter tests (Figure S6) and survived cells allowing BE activity studies. Although direct comparison with earlier reports is not possible due to earlier mentioned parameters, these findings demonstrate the robust applicability of the IRI-CCE platform to investigate features of broad-range CBE tools.

A

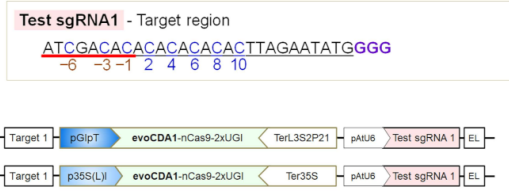

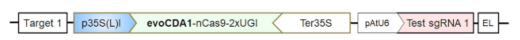

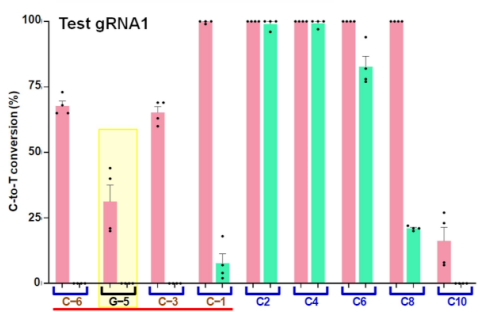

C

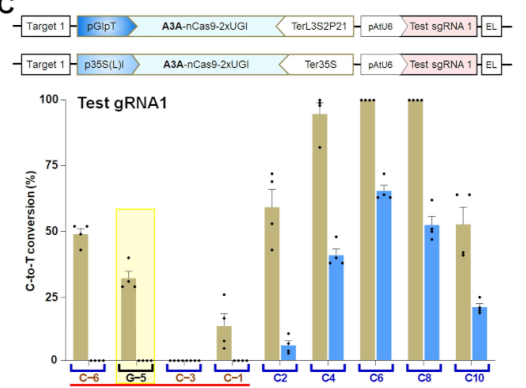

Test sgRNA2 - Target region
$\frac{\text { ATCGACACCACACACACATTAGAATATGGGG }}{-6-3-11359}$

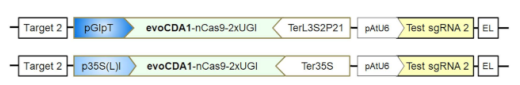

$\left.{ }^{100}\right]$ Test gRNA2
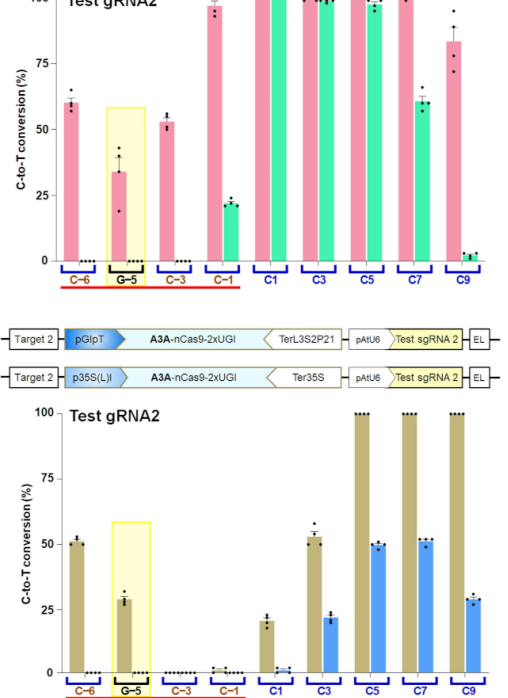

Test sgRNA3 - Target region $\frac{\text { ATCGACACAAACCCCTCCCCCCACCAGCCGG }}{-6-3-1} \frac{456791011213141617}{456}$
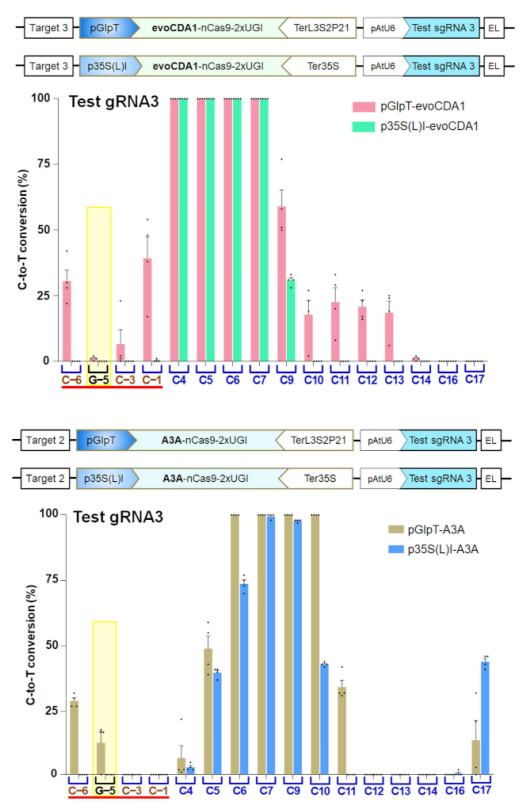

Figure 6. The evoCDA1 and APOBEC3A expressed under the control of different promoters show expanded editing windows with efficient C-to-T editing. (A) Nucleotide sequences of three sgRNAs (underlined in black) along with additional $N$-terminal regions (underlined in red). The numbers are assigned by counting the distal base as 1 from the protospacer adjacent motif (PAM), i.e., GGG. $(B, C)$ evoCDA1 (B) and APOBEC3A (A3A) (C) driven C-to-T editing activities for three substrates targeted in three independent experiments. The designed BE constructs are represented on the upper side and obtained results are depicted in bar graphs. G-5 position (yellow shade) data showed G.C to A.T conversion, suggesting editing happened in the target strand. Graph values show the mean percentage on the $y$-axis and the tested protospacer positions on the $x$-axis. The graph bar shows the mean of percentage values, and error bars indicate the standard error of the mean (mean \pm s.e.m.) of four independent biological replicates. Dots indicate the individual biological replicates.

\subsection{ABE8e and ABE9e Activities for A-to-G Conversion in E. coli}

Recently, evolved ABE variants, TadA-8e (ABE8e) and TadA-9e (ABE9e), have been characterized and have shown improved A-to-G base conversion in vitro, in humans, and in plants, respectively $[20,21]$, but have not yet been systematically evaluated in bacteria. The sgRNAs with the multiple As in a target region exhibited different A-to-G editing efficiencies for each position in E. coli and S. aureus [22]. Moreover, the editing window for $\mathrm{ABE}$ was also reported to vary with the target site, host species, genotype, and experimental conditions $[7,35]$. We sought to investigate the ABE8e and ABE9e activity using two synthetic sgRNAs (Figure 7). The editing window for ABE8e and ABE9e variants 
was reported as positions 4 to 8 in human cells [20] and 1 to 11 in plants [21], respectively. Editing levels of ABE8e and ABE9e showed significant increases in the case of pGlpT compared to that of p35S(L)I (Figure 7A-C).

A

B
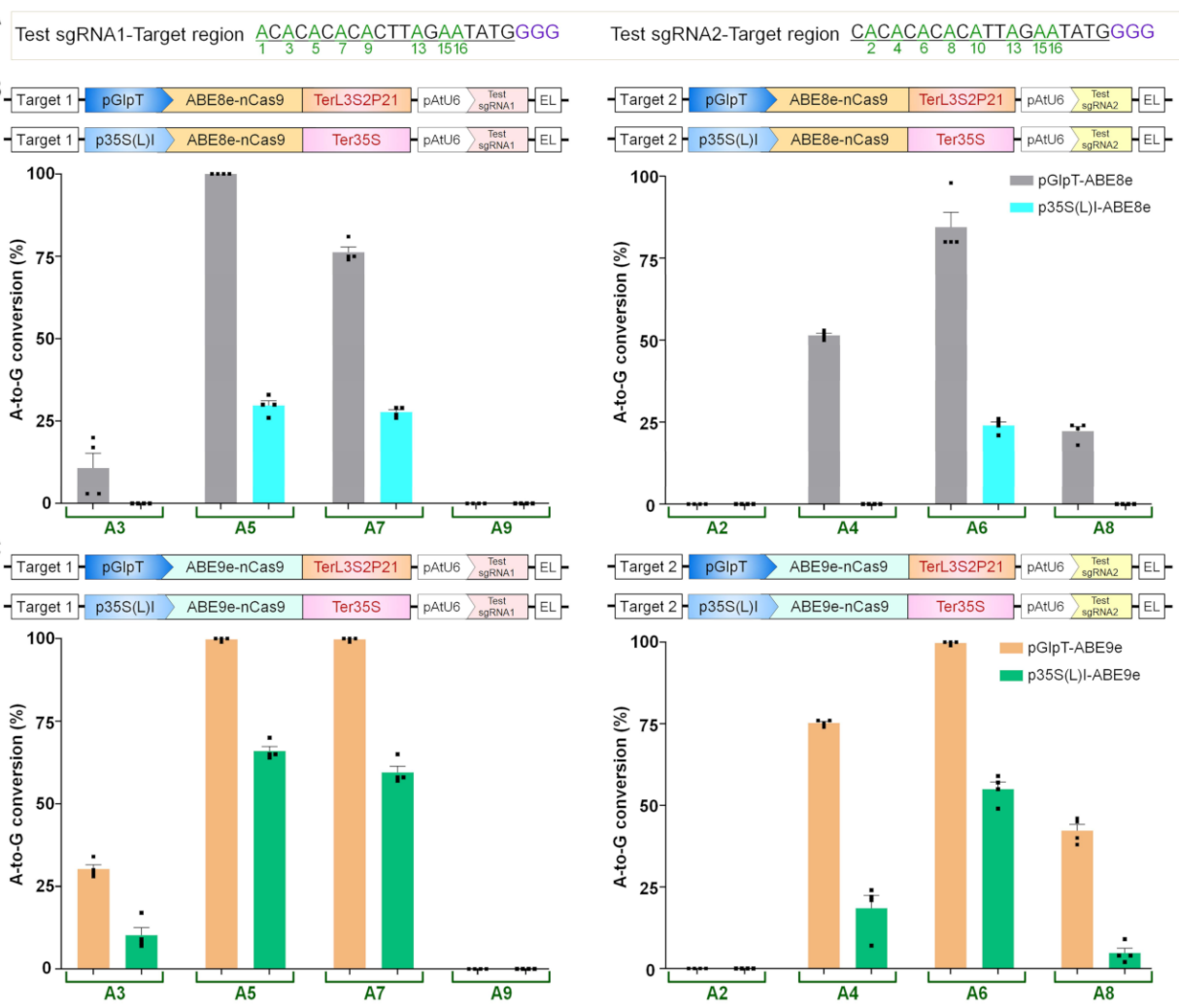

Figure 7. Adenine base editor variants (ABE8e and ABE9e) efficiently convert A to G in E. coli cells. (A) Nucleotide sequences of Test sgRNA1 and -2 (underlined in black) showing available adenine (A) residues (green). (B,C) The A-to-G conversion efficiencies of ABE8e (B) and ABE9e (C) driven by pGlpT and p35S(L)I promoters in the target region for Test sgRNA1 and Test sgRNA2. The graph bar shows the mean of percentage values, and error bars indicate the standard error of the mean (mean \pm s.e.m.) of four independent biological replicates. Dots indicate the individual biological replicates.

We observed the editing window of ABE8e from positions 3 to 8 and 5 to 7 for pGlpT and p35S(L)I, respectively. The editing window of ABE9e for pGlpT and p35S(L)I ranged from positions 3 to 8 and 3 to 7 , respectively. A-to-G conversion at positions 5 to 7 was the highest in the case of both the variants (ABE8e: 74-100\%; ABE9e: 99-100\%). For p35S(L)IABE8e, A-to-G mutation at positions 5 to 7 was the highest but in the lower range (21-33\%), and $\mathrm{p} 35 \mathrm{~S}(\mathrm{~L}) \mathrm{I}-\mathrm{ABE}$ e showed a moderate editing activity (55-66\%). These data suggest that the editing activity of ABE9e was more enriched than that of ABE8e in E. coli, and editing activity increases in a promoter-strength-dependent manner. In the case of ABEs, editing activity outside the gRNA window was not observed. Overall, all CBE and ABE data indicate that the differential-strength promoters may contribute to the variable editing outcomes within the canonical editing window.

\subsection{IRI-CCE Platform Allows Screening of Functional sgRNAs}

Prescreening of sgRNAs before commencing GE studies is crucial in higher eukaryotes to avoid loss of time for sgRNA screening, lower editing activities, and waste of resources. Given that the efficiencies predicted by in silico tools and the actual GE activities may differ significantly, in vitro or preferentially in vivo validation of chosen sgRNAs is vital before the actual tests [36]. Generally, protoplast and transient agroinfiltration assays are used for preliminary screening in plants, with some limitations elaborated in the discussion 
section. In that scenario, the IRI-CCE platform may complement these existing platforms, allowing the simultaneous evaluation of designed bioparts and functionality of the selected sgRNAs that enable the potential avoidance of incorrect plasmids and inefficient gRNAs. In some instances, sgRNAs predicted as inactive by bioinformatic tools or design criteria were reported to work efficiently under in vivo conditions [37]. On the other hand, even if in silico parameters are not promising for a particular set of sgRNAs, one needs to choose those sgRNAs for editing a specific region of targeted locus due to PAM constraints.

We analyzed sets of active and inactive sgRNAs reported in the previous studies (Figure $8 \mathrm{~A}$ ). Based on the $\mathrm{A}$ or $\mathrm{C}$ availability in the canonical editing window, we opted for a particular BE type to edit the target sites of individual sgRNAs. The target regions, including the PAM, were cloned using two BsmBI type IIS sites in the universal targetacceptor modules. Firstly, the set of three active sgRNAs (AtGL1-sgRNA1, [38] SIMlo1sgRNA2, and SIPelo-sgRNA1 [39]) targeting three genomic targets showed higher base conversion efficiencies (Figure 8B-D) with differential-strength promoters as observed under in vivo conditions.

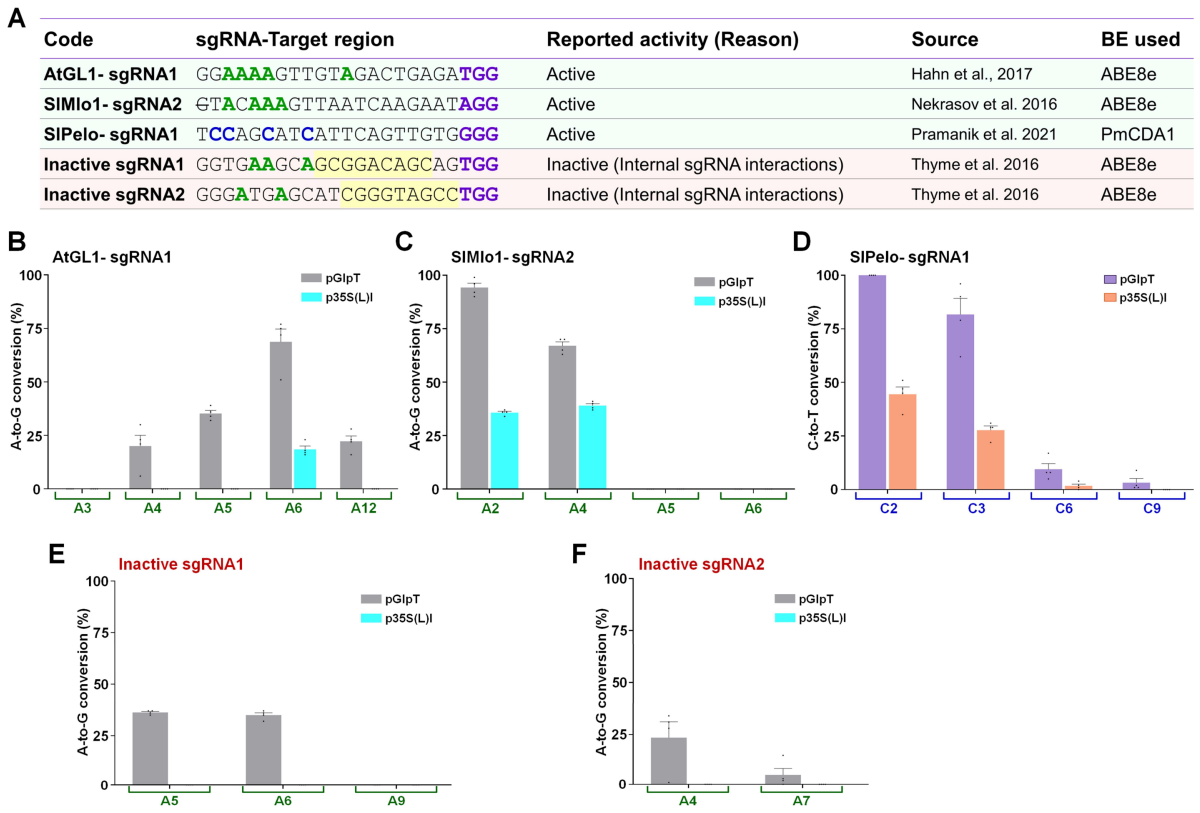

Figure 8. Screening of designed sgRNAs in the IRI-CCE platform provides first-hand knowledge about the sgRNA functionality. (A) Set of sgRNAs used for validating the potential use of IRI-CCE in sgRNA screening. The base editor (BE) type was chosen depending on the availability of A or $\mathrm{C}$ in the editing window. Frequencies of base conversions by utilized BE types in target regions of AtGL1-sgRNA1 (B), SIMlo1-sgRNA2 (C), and SlPelo-sgRNA1 (D), Inactive sgRNA1 (E), and Inactive sgRNA2 (F). The graph bar shows the mean of percentage values, and error bars indicate the standard error of the mean (mean \pm s.e.m.) of four independent biological replicates. Dots indicate the individual biological replicates.

Secondly, we analyzed ABE8e activities for two inactive sgRNAs (Inactive sgRNA1 and Inactive sgRNA2) that displayed poorer performance [40]. The lower editing activity was attributed to internal sgRNA interactions that allow binding with Cas9, but it prevents target DNA recognition by the Cas9-sgRNA complex (Figure S8). Besides, inactive sgRNA competes with active sgRNAs for binding to the Cas9 protein, thereby reducing the editing activities. We investigated the base conversion activity at target sites of both the sgRNAs and discovered that both the sgRNAs led to no BE frequencies in the target DNA region with low-strength p35S(L)I (Figure 8E,F), consistent with the in vivo data for CRISPR/Cas editing [40]. Moreover, even a high-strength promoter (pGlpT)-driven ABE8e expression led to relatively lower BE activity ( $36 \%$ and $23.5 \%$ for Inactive sgRNA1 and -2 , respectively). 
Overall, these data indicate that the IRI-CCE platform is helpful to distinguish the functional sgRNAs, although with limited information (elaborated in the discussion section). Taken together, the IRI-CCE platform could still be an asset to provide first-hand knowledge of in vivo sgRNA activity one needs to further validate or use in higher eukaryotes. Especially, IRI-CCE may be more sensitive than in planta methods and could be helpful in screening gRNAs for plant species without readily available protoplast or agroinfiltration systems.

\subsection{Expanding the Targeting Scope of BEs by Altering Editable Window Size Using Different Lengths of Protospacers}

Different lengths of protospacers have been reported to alter the editing window of CBE versions in the case of plasmid and chromosomal targets [9,41,42]. To further characterize the effect of protospacer length together with promoter strength on editing window shift, protospacers 18, 20, 21, 22, 23, 25, and $30 \mathrm{nt}$ in length were tested for CBE (Target-AID, Figure 9A) and ABE (ABE9e, Figure 9B). A narrow editing window was observed for truncated protospacer (18-nt) compared to conventional 20-nt protospacer. The editable window was shifted towards the $3^{\prime}$-end of the target sequence, i.e., towards the PAM site (counting PAM as 21 to 23). In contrast, sgRNAs with extended length consistently showed window shift and expansion of the editable window towards the $5^{\prime}$ end of the target sequences in the case of both the promoter types (Figure 9).

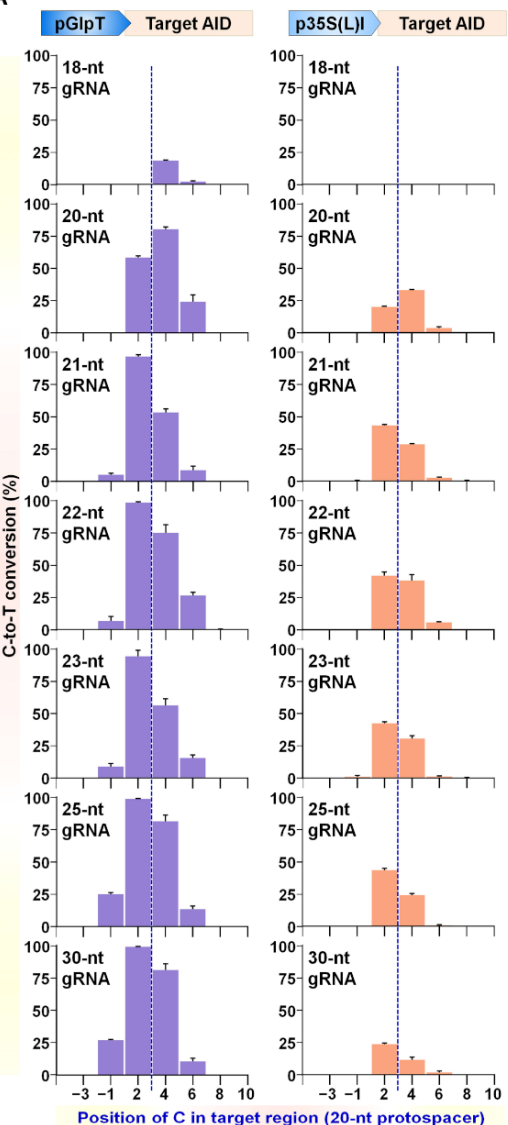

B

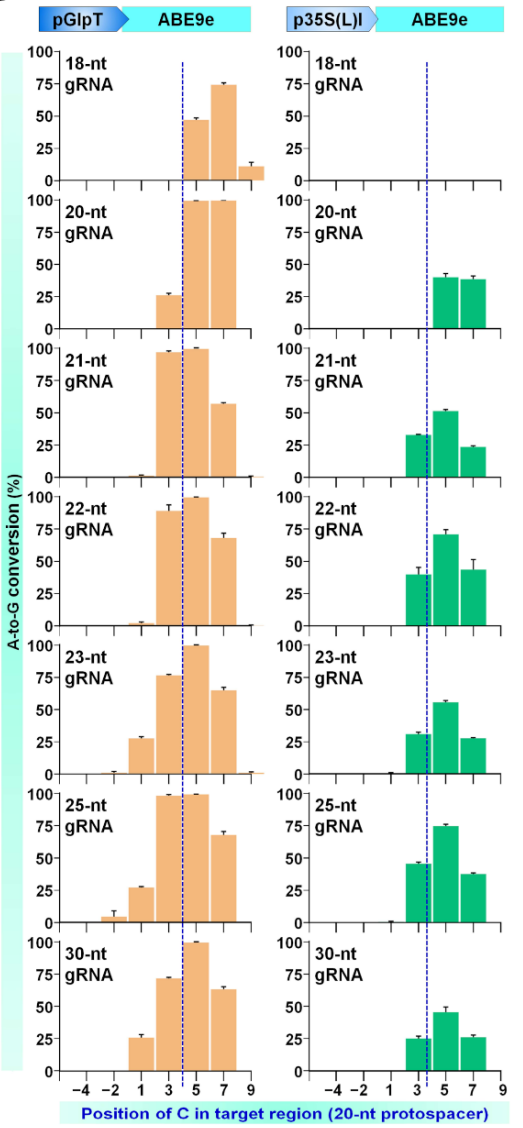

Figure 9. Effect of gRNA length on editing window length in Target-AID and ABE9e. Base editor (BE) efficiencies were tested in the target region by different lengths for Test sgRNA1 (18, 20, 21, 22, 23, 25, 30 nucleotides). Base conversion from C to T with cytosine BE variant Target-AID (A) and from A to $\mathrm{G}$ with adenine BE variant ABE9e (B) driven by pGlpT and p35S(L)I promoters plotted in graphs. The graph bar shows the mean of percentage values of four independent biological replicates. 
As shown in Figure 6, broad-range CBEs, particularly evoCDA1, exhibited higher C-to-T conversion with a wider editing window in 20-nt protospacer (pGlpT: positions -6 to 14, p35S(L)I: positions -1 to 9). Likewise, higher editing was observed in all the tested protospacers of different lengths for pGlpT and p35S(L)I promoters for evoCDA1 (Figure 10). Particularly at -1 and -3 positions for p35S(L)I, base editing was about 4 -fold and 30-fold higher than with a 20-nt protospacer. With a longer length of protospacers (21, 22,23 , and $25 \mathrm{nt}$ ), $\mathrm{C}$ at position -6 was edited (up to 19\%), which was unchanged in 20-nt protospacer for p35S(L)I. Interestingly, 30-nt protospacer was found to be less active in all three BE versions. A similar trend was reported for protospacers with lengths of more than $25 \mathrm{nt}$ [41]. Overall, BE tools showed narrow or wider editable windows in the case of differential-strength promoters together with extended or truncated protospacers.

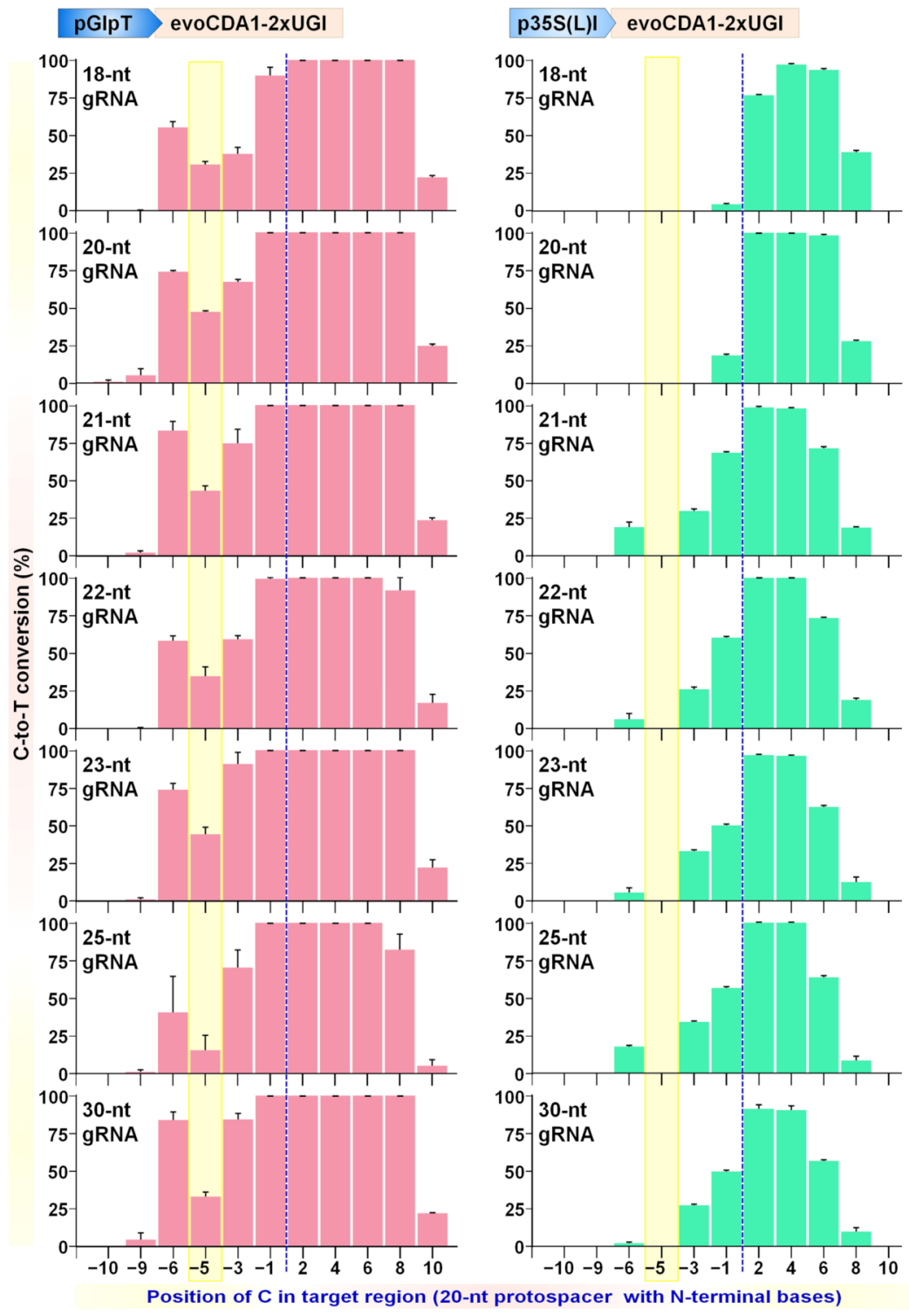

Figure 10. The gRNA lengths alter editing window size in broad-range cytosine base editor evoCDA1. Cytosine base editor variant evoCDA1 expressed using pGlpT and p35S(L)I promoters for C-to-T editing in the target region by different lengths for Test gRNA1 (18, 20, 21, 22, 23, 25, 30 nucleotides). The G-5 position (yellow shade) data showed G.C to A.T conversion, suggesting C-to-T editing happened in the target strand. The graph bar shows the mean of percentage values of four independent biological replicates. 


\section{Discussion}

Several features of E. coli make it a model microorganism in molecular biology. These include a short life cycle, simple genetic manipulations, low cost, and in-depth knowledge of the genome [43]. In the current study, we developed an E. coli-based IRI-CCE platform to investigate important aspects, including optimization of $\mathrm{BE}$ tools, validation of $\mathrm{BE}$ bioparts for further use in modular cloning approach, and sgRNA functionality with several advantages. IRI-CCE enables the rapid verification of designed BE bioparts. Modular bioparts are exchangeable for cloning and investigating features of different CRISPR-based $\mathrm{BE}$ tools in bacteria and plants, including deaminase variants, Cas 9 forms, heterologous promoters (p35S and pAtU6), and accessory components such as UGI. Moreover, the approach reported here could be readily scaled to many constructs and different BE types and evolved versions. In addition, multiple gRNA and DNA targets can be evaluated by using modular cloning assembly in the future. In addition, IRI-CCE could also be useful for testing novel $\mathrm{BE}$ architectures.

The attempt to analyze the BE activity of bacterial (pJ23119) promoter-driven sgRNA expression was unsuccessful due to the toxicity caused by BE components. Likewise, the pEc1-mediated CBE test also showed poor transformation efficiency with a mix of incorrect clones. The higher expression of $\mathrm{BE}$ components adversely affects cell survival in bacteria $[9,14]$. When $\mathrm{nCas9}(\mathrm{D} 10 \mathrm{~A})-\mathrm{PmCDA1} 1 \times \mathrm{UGI}$ was expressed together with pJ23119-sgRNA, no correct clones were obtained in E. coli, possibly due to toxic effects of BE components [9]. However, nCas9(H840A)-PmCDA1 did not lower the transformation efficiency, suggesting that the SSBs induced by nCas9(H840A) may be insufficient to cause cell toxicity. Instead, more likely, the transient DSBs generated by two nicks on different strands by $\mathrm{nCas} 9(\mathrm{D} 10 \mathrm{~A})-\mathrm{PmCDA}$ (one on the non-deaminated strand by $\mathrm{nCas} 9(\mathrm{D} 10 \mathrm{~A}$ ) and the other on the deaminated strand by AP endonuclease during the repair process) may lead to cell toxicity. A high rate of SSBs and transient DSBs induced by nCas9(D10A)-PmCDA1 during pJ23119-mediated sgRNA production seems challenging to restore by the bacterial system, ultimately resulting in losing the plasmid. The cloning of dCas9-PmCDA1-1 $\times$ UGI with pJ23119-sgRNA showed the wrong clones in E. coli [9], suggesting cells cannot retain the functional dCas9-PmCDA1-1 $\times$ UGI-expressing plasmids. Although dCas9 does not introduce SSBs or transient DSBs, earlier dCas9-PmCDA1-1 $\times$ UGI data indicated that the surplus amount of UGI compromises the genome integrity in CBE types [9].

Previous studies reported that the use of protein degradation (LVA) tag fusion with dCas9-Target-AID reduced the toxicity to E. coli cells [9]. Producing the optimal amount of $\mathrm{BE}$ and sgRNA might reduce the toxicity to a tolerable level for a bacterial cell, as observed in nCas9-BE together with pAtU6-driven sgRNA expression. As described in our study, the combination of pGlpT-nCas9-BE or p35S(L)I-nCas9-BE with pAtU6-sgRNA expression units proved to be the most appropriate to edit the target site in the same plasmid vector or E. coli genome without causing cytotoxicity. Therefore, fine-tuning the expression of BEs and sgRNAs by using the optimal combination of promoters is a crucial factor in minimizing the toxic effects in the IRI-CCE platform. A recent study [44] indicated that the sufficiently perturbed dsDNA in bacteria-during replication, transcription, or other rearrangements-provides enough opportunity for Cas9 binding to a target site in the form of dynamically stretched single-strand DNA (ssDNA) or un- and underwound doublestrand DNA. Therefore, it is conceivable that the fewer nCas9-BE enzyme molecules, mainly evolved versions with a faster catalytic rate $[18,45]$, produced by p35S-based promoters are enough to show relatively higher deamination activity.

Deaminase interaction with Cas protein and target DNA region alters the location and size of the editing window [46]. The indispensable role of the endogenous DNA repair mechanism is a decisive factor that may produce variable BE results in different organisms [47]. Nevertheless, we observed significant differences between the targeting competencies of three CBEs owing to the differential strength of promoters used for Cas-BE expression (Figure S9). The evoCDA1 is an evolved variant of PmCDA1 [18] that showed higher catalytic activity and expanded editing window than its wild-type counterpart. We 
observed higher C-to-T editing in the expanded window up to position -6 that was not detected in the previous work for evoCDA1 or A3A. It has been reported that different lengths of gRNA enable the adjustment of the editing window in BE tools [9,41,42]. Likewise, tested $\mathrm{BE}$ tools showed narrow or wider editing windows in the case of differential-strength promoters and extended or truncated gRNAs (Figures 9 and 10). Therefore, we propose using a combination of variable lengths of sgRNAs, differential expression of BE components, and engineered Cas9 variants with relaxed PAM sites as a new approach to adjust the editing window and targeting scope of BE tools.

Although we consistently observed differences in the editing window size for all the tested BE versions under differential-strength promoters (Figure S9), the exact mechanism is unclear. We note that a recent report by Hao and coworkers [41] has analyzed the effects of variable expression levels of nCas9-PmCDA1 on conversion efficiency and editing window in B. subtilis. Higher base editing (C-to-T) efficiency and wider editing window were exhibited with an increased concentration of inducer in the case of xylose promoter $\left(\mathrm{P}_{\mathrm{xyl}}\right)$. Notably, the most preferred position is the same for specific $\mathrm{BE}$ versions irrespective of the promoter type (PmCDA1, position 4; evoCDA1, positions 2 to 7; APOBEC3A, positions 6 to 8; ABE8e, positions 5 to 7; ABE9e, positions 5 to 7). Nucleotide conversion efficiency on both sides of the most preferred position showed a gradual decrease in all tested promoter-BE combinations, consistent with previous reports about enzymatic activities of deaminases $[45,48,49]$. Indeed, the interplay between various factors related to enzyme-substrate concentrations (nCas9-BE, ssDNA) inside the cell may contribute to the final BE outcome. Some key aspects involve the rate of DNA replication, target DNA packaging and accessibility, amount of nCas9-BE, the kinetics of deamination, and DNA repair of deaminated nucleotides [48,50]. Further studies are needed to elucidate the role of these factors in $\mathrm{BE}$ outcomes. We believe that further comprehensive analysis of the promoter-strength-dependent variability in the editing window will help to identify the factors responsible for wider or narrow editing windows.

Some studies reported C-to-G or C-to-A as CBE editing byproducts [5,8]; we did not observe this phenomenon in the case of basic (PmCDA1, APOBEC3A) or improved CBE (evoCDA1) versions, which might be too low to detect with Sanger sequencing if present. The evoCDA1 and APOBEC3A exhibited different editing windows, possibly due to differences in editing competencies of deaminases and target accessibility during R-loop formations. Some Cs in the editing window were not accessible to APOBEC3A deamination. These observations highlight the importance of considering BE type and promoter strength that best suit the expected outcome. Moreover, temperature affects the enzymatic activities of CRISPR components, thereby influencing the GE outcome [2]. Mainly, the performance of Cas enzymes is best at $37^{\circ} \mathrm{C}$. So, besides the promoter activity and evolved variants, better editing efficacy in IRI-CCE may result from the favorable temperatures for enzymatic activities.

The IRI-CCE system in its present form has some limitations related to validating plant-related factors while optimizing BE modules or gRNA screening. Some plant-related factors cannot be assessed in this bacteria-based IRI-CCE system for reasons that include the effect of genome organization on target accessibility, the feature to evaluate nuclear localization signal (NLS) function (although NLSs are included in the expression modules), different DNA repair machinery in bacteria, and difference in growth temperature of bacteria and plants. However, IRI-CCE offers an efficient platform for bacterial BE studies, evidenced by its successful use for optimizing different nCas9-based BE versions (PmCDA1, evoCDA1, APOBEC3A, ABE8e, and ABE9e), which is also the first report in E. coli to the best of our knowledge. In addition, IRI-CCE allows verification of the sgRNA-related factors such as nCas9-BE-sgRNA complex formation and target DNA-binding irrespective of the host system. Some existing platforms can be helpful for prescreening of gRNAs and preliminary check of CRISPR-BE outcomes. For instance, the combination of protoplast culture and preassembled ribonucleoprotein (RNP) complexes is one of the handy methods for assessing the editing activities of sgRNAs in plants [51]. 
Protoplast systems are relatively easy to use as testbeds for GE tests. A growing number of protocols are being established for protoplast isolation and RNP-mediated delivery of CRISPR reagents in many plant species, including model and nonmodel plants [52-56]. Nevertheless, the prerequisite of protoplast-based assays is the establishment of optimized protoplast isolation procedures for the crop of interest and purified Cas-sgRNA RNP complexes, which further adds to the cost. Transient agroinfiltration assay proved to be a comparatively quick in vivo method for analyzing sgRNA efficacy, but the lower activity and inconsistency depending on target plant species are the significant obstacles [57]. The IRI-CCE platform may serve as a fast way to validate some key aspects. For instance, the sgRNA-related factors such as nCas9-BE-sgRNA complex formation or stability and target DNA-binding would be identified in the IRI-CCE platform. We propose that the users choose the moderate $\mathrm{p} 35(\mathrm{~L}) \mathrm{I}$ promoter in sgRNA prescreening experiments to avoid overestimating sgRNA activity while using the pGlpT promoter. Moreover, the use of a specific BE type depending on the nucleotide composition of the target region is recommended. However, more efforts are needed to understand uncovered aspects. For example, the editing efficiency of each nCas9-BE-sgRNA complex differs significantly depending on the target locus [39]. In higher eukaryotes, editing differences arise from the sequence bias in DNA repair machinery and target accessibility [58]. Moreover, the bigger genome size of eukaryotes influences the dynamics of target search. Therefore, IRI-CCE is missing those features common to eukaryotic cells and cannot be verified in the current platform. While our data show the promising way to confirm the biopart modules for CRISPR-based BE work, there are multiple opportunities to expand the IRI-CCE utility further in other bacteria. Moreover, the design and adoption of new dual BEs by combining different variants of $\mathrm{ABE}$ and $\mathrm{CBE}$ could be interesting for simultaneous conversion of $\mathrm{A}$ to $\mathrm{G}$ and $\mathrm{C}$ to $\mathrm{T}$, respectively.

\section{Materials and Methods}

\subsection{E. coli Strains}

The following four E. coli strains were used: 10-beta, DH5 $\alpha$, DB3.1, and BL21(DE3). The information about strain genotype is provided in Table S2. The primers, plasmids, and biopart sequences used in this study are listed in Tables S3-S5, respectively. The E. coli strains were cultured in Luria-Bertani (LB) broth with appropriate antibiotics.

\subsection{Plasmid Construction and Cloning}

The required bioparts were amplified by conventional polymerase chain reaction (PCR) using a Phusion high-fidelity DNA polymerase (Thermo Fisher Scientific, Waltham, MA, USA). The cloning of different plasmid vectors was designed and performed by following the principle of MoClo [59] and Golden Gate assembly [17] protocols using BpiI/BsaI Type IIS enzyme digestion-ligation. The DNA recognition sites of restriction enzymes BsaI and BpiI were removed from internal sequences of bioparts during PCR amplification to make them suitable for Golden Gate cloning (a procedure also termed domestication). The DNA oligonucleotide pairs for the pEc1 (SJM901 + RBSTL2) promoter and TerL3S2P21 terminator were annealed and ligated into BpiI-digested acceptor plasmids. Other promoters including pGlpT and p35S(L)I were used from the pYTK001 (Addgene \#65108) [25] and pICH51266 (Addgene \#50267) [17], respectively. The promoter DNA sequences and their evaluation are provided in Figures S1-S3. Similarly, DNA sequences of Ter35S Terminator were adopted from the pICH41414 (Addgene \#50337) [17].

Three cytidine deaminases, namely PmCDA1 (Target-AID) [6], evoCDA1 [18], and APOBEC3A [19], were PCR amplified from PmCDA1-1×UGI (Addgene \#79620), evoCDA1 pBT277 (Addgene \#122608), and A3A-PBE-AUGI (Addgene \#119770), respectively. For all three deaminases, the same linker regions were used as reported in the previous studies. The ABE8e was synthesized from Bioneer Co. (Daejon, Korea), and the ABE9e variant (containing additional V82S/Q154R mutations) was cloned using ABE8e module as a template by site-directed mutagenesis PCR. PmCDA1-1 $\times$ UGI was fused to the $C$ terminus 
of nCas9. The evoCDA1, A3A, and ABE8e were fused to the $\mathrm{N}$ terminus of $\mathrm{nCas} 9$ with the XTEN linker. The $2 \times$ UGI module (template: Addgene \#122608) was fused to the C terminus of evoCDA1-nCas9 and A3A-nCas9. The sgRNA expression was driven by bacterial pJ23119 (synthetically cloned) or plant AtU6 promoter (pICSL01009, Addgene \#46968). The nCas9(D10A) was generated by the PCR method using previously optimized Cas9 as a template (Level 1 hCas9 module, Addgene \#49771). Desired sgRNA sequence was PCR amplified using plasmid pICH86966::AtU6p::sgRNA_PDS (Addgene \#46966) as a template and cloned together with either pJ23119 or pAtU6 for sgRNA expression. All the Addgene constructs ordered from https://www.addgene.org/, accessed on 9 December 2021.

For cloning of the target region of the desired sgRNA sequence, a pair of oligonucleotide DNAs that contained the target sequence with PAM was annealed and ligated into BsmBI-digested universal target-acceptor plasmid L1 or L2 having optimized sfGFP [25] at downstream side (Figures 1 and 2).

\subsection{Bacterial Transformation, Plasmid Isolation, and Sanger Sequencing}

Ligation products of all the steps during the cloning were transformed into competent cells of $E$. coli 10-beta strain by a heat-shock method. The bacterial culture was spread on the LB media containing desired antibiotic and incubated at $37^{\circ} \mathrm{C}$ for $18-24 \mathrm{~h}$. The cloned plasmids and BE activities were confirmed by Sanger sequencing at Solgent Ltd. (Daejeon, Korea) or Cosmogentech Ltd. (Seoul, Korea). For mutagenesis assay, the different E. coli strains were transformed with the appropriate plasmids using the heat-shock method and were precultured for $1 \mathrm{~h}$ with $1 \mathrm{~mL}$ of LB medium. After incubation for $1 \mathrm{~h}$ at $37^{\circ} \mathrm{C}$, the fraction of cell cultures were spread on LB agar $(1.5 \%)$ plates containing selection antibiotics with needed concentrations. The next day, individual colonies from the plate were inoculated in $3 \mathrm{~mL}$ LB broth with appropriate antibiotics and cultured at $37^{\circ} \mathrm{C}$. The culture time was varied according to the experimental parameters and mentioned in appropriate sections. The plasmid isolation was performed using Plasmid Mini-Prep Kit from BioFact Co. Ltd. (Daejeon, Korea) for Sanger sequencing analysis.

\subsection{Promoter Activity Analysis}

RNA was extracted using the following method: E. coli-carrying plasmids (Figure 1B) were grown in LB medium. Cells were harvested during the exponential growth phase (12 h with $\mathrm{OD}_{600}$ value 0.5 ). Total RNA was extracted using RNeasy Protect Bacteria Mini Kit from Qiagen. For all samples, $600 \mathrm{ng}$ of total RNA was used for complementary DNA (cDNA) synthesis using a QuantiTect Reverse Transcription Kit from Qiagen following the manufacturer's instructions. To estimate the relative sfGFP transcript, the quantitative real-time PCR (qRT-PCR) reactions were carried out using the KAPA SYBR FAST qPCR kit from Kapa Biosystems (Wilmington, MA, USA) with sfGFP-specific primer sets (Table S5). Cycling of PCR consisted of pre-denaturation at $95^{\circ} \mathrm{C}$ for $5 \mathrm{~min}$ followed by 40 cycles of a denaturation step at $95^{\circ} \mathrm{C}$ for $10 \mathrm{~min}$, an annealing step at $60^{\circ} \mathrm{C}$ for $15 \mathrm{~s}$, and final extension step at $72{ }^{\circ} \mathrm{C}$ for $20 \mathrm{~s}$ using the CFX384 Real-Time System from Bio-Rad (Hercules, CA, USA). The qRT-PCR reactions were performed with independent biological replicates. Relative $s f G F P$ transcript values normalized against internal control $16 \mathrm{~S}$ ribosomal RNA ( $r r s A)$ gene. Data analyses were performed by the $2^{-\Delta \Delta C t}$ method [60].

\subsection{Evaluation of Editing Activities}

The single colonies were cultured, and the plasmid vectors containing synthetic targets were purified using Plasmid Mini-Prep Kit from BioFact Co. Ltd. (Daejeon, Korea) for further sequencing analysis. For sequencing analysis of genomic loci, the target fragments were PCR amplified using target-specific primers from the randomly picked colonies and then analyzed by Sanger sequencing. Sanger sequencing data analysis was performed using SnapGene software version 3.2.1 (GSL Biotech; available at snapgene.com, accessed on 9 December 2021). The editing efficiency was determined by the ratio of nonedited to 
edited colonies from the randomly picked cells. The base conversion rate was estimated using the online tool EditR [61] from $C$ to $T$ and $A$ to $G$ for CBE and ABE, respectively. The data were statistically analyzed and plotted in GraphPad Prism software version 9.0.0 for windows from GraphPad Software, San Diego, CA, USA (www.graphpad.com, accessed on 9 December 2021).

\section{Conclusions}

In summary, nCas9-BE components expressed by promoters of different strengths led to the establishment of the nontoxic IRI-CCE platform for two major applications: investigating cloned CRISPR-BE components and understanding sgRNA functionality. IRI-CCE analyses showed the variable length of the editing window for specific BE types. Each independent BE type has its specific editing pattern for a given target site and promoter type. Because of its easy cloning and heterologous applicability, the IRI-CCE platform may be suited for rapid evaluation of cloned bioparts in modular cloning of BE studies and applicable to a broader range of $E$. coli strains being used for cloning. This platform facilitates the verification of sgRNA-related factors such as cloning, sgRNA production, Cas9-sgRNA complex formation, and DNA recognition. Through the applications described here and through further improvements, IRI-CCE can be widely applicable for the characterization of BE components and rapid assessment of newer CRISPR-based BE technologies.

Supplementary Materials: The following are available online at https:/ / www.mdpi.com/article/10 $.3390 /$ ijms23031145/s1.

Author Contributions: Conceptualization R.M.S. and J.-Y.K.; methodology, R.M.S.; validation, R.M.S. and D.P.; formal analysis, R.M.S., D.P. and J.-Y.K.; investigation, R.M.S. and D.P.; resources J.-Y.K.; writing-original draft preparation, R.M.S.; writing-review and editing, R.M.S., D.P. and J.-Y.K.; visualization, R.M.S. and D.P.; supervision, J.-Y.K.; project administration, J.-Y.K.; funding acquisition, R.M.S. and J.-Y.K. All authors have read and agreed to the published version of the manuscript.

Funding: This work was supported by the National Research Foundation of Korea (grants NRF 2020M3A9I4038352, 2021R1A5A8029490, 2021R1I1A3057067) and the Program for New Plant Breeding Techniques (NBT, grant PJ01478401), Rural Development Administration, Korea.

Institutional Review Board Statement: Not applicable.

Informed Consent Statement: Not applicable.

Data Availability Statement: All datasets supporting the conclusions of this article are included in the article and supplementary files.

Conflicts of Interest: The authors declare no conflict of interest.

\section{References}

1. Anzalone, A.V.; Koblan, L.W.; Liu, D.R. Genome editing with CRISPR-Cas nucleases, base editors, transposases and prime editors Nat. Biotechnol. 2020, 38, 824-844. [CrossRef]

2. Pramanik, D.; Shelake, R.M.; Kim, M.J.; Kim, J.-Y. CRISPR-mediated engineering across the central dogma in plant biology for basic research and crop improvement. Mol. Plant 2021, 14, 127-150. [CrossRef] [PubMed]

3. Tong, Y.; Jørgensen, T.S.; Whitford, C.M.; Weber, T.; Lee, S.Y. A versatile genetic engineering toolkit for E. coli based on CRISPRprime editing. Nat. Commun. 2021, 12, 5206. [CrossRef] [PubMed]

4. Ding, W.; Zhang, Y.; Shi, S. Development and application of CRISPR/Cas in microbial biotechnology. Front. Bioeng. Biotechnol. 2020, 8, 711. [CrossRef] [PubMed]

5. Komor, A.C.; Kim, Y.B.; Packer, M.S.; Zuris, J.A.; Liu, D.R. Programmable editing of a target base in genomic DNA without double-stranded DNA cleavage. Nature 2016, 533, 420-424. [CrossRef]

6. Nishida, K.; Arazoe, T.; Yachie, N.; Banno, S.; Kakimoto, M.; Tabata, M.; Mochizuki, M.; Miyabe, A.; Araki, M.; Hara, K.Y.; et al. Targeted nucleotide editing using hybrid prokaryotic and vertebrate adaptive immune systems. Science 2016, 353, aaf8729. [CrossRef]

7. Huang, T.P.; Newby, G.A.; Liu, D.R. Precision genome editing using cytosine and adenine base editors in mammalian cells. Nat. Protoc. 2021, 16, 1089-1128. [CrossRef]

8. Rees, H.A.; Liu, D.R. Base editing: Precision chemistry on the genome and transcriptome of living cells. Nat. Rev. Genet. 2018, 19, 770-788. [CrossRef] 
9. Banno, S.; Nishida, K.; Arazoe, T.; Mitsunobu, H.; Kondo, A. Deaminase-mediated multiplex genome editing in Escherichia coli. Nat. Microbiol. 2018, 3, 423-429. [CrossRef]

10. Sun, J.; Lu, L.-B.; Liang, T.-X.; Yang, L.-R.; Wu, J.-P. CRISPR-assisted multiplex base editing system in Pseudomonas putida KT2440. Front. Bioeng. Biotechnol. 2020, 8, 905. [CrossRef]

11. Yu, S.; Price, M.A.; Wang, Y.; Liu, Y.; Guo, Y.; Ni, X.; Rosser, S.J.; Bi, C.; Wang, M. CRISPR-dCas9 mediated cytosine deaminase base editing in Bacillus subtilis. ACS Synth. Biol. 2020, 9, 1781-1789. [CrossRef]

12. Kim, M.S.; Kim, H.-R.; Jeong, D.-E.; Choi, S.-K. Cytosine Base editor-mediated multiplex genome editing to accelerate discovery of novel antibiotics in Bacillus subtilis and Paenibacillus polymyxa. Front. Microbiol. 2021, 12, 691839. [CrossRef] [PubMed]

13. Zhao, Y.; Tian, J.; Zheng, G.; Chen, J.; Sun, C.; Yang, Z.; Zimin, A.A.; Jiang, W.; Deng, Z.; Wang, Z.; et al. Multiplex genome editing using a dCas9-cytidine deaminase fusion in Streptomyces. Sci. China Life Sci. 2020, 63, 1053-1062. [CrossRef] [PubMed]

14. Rodrigues, S.D.; Karimi, M.; Impens, L.; Van Lerberge, E.; Coussens, G.; Aesaert, S.; Rombaut, D.; Holtappels, D.; Ibrahim, H.M.M.; Van Montagu, M.; et al. Efficient CRISPR-mediated base editing in Agrobacterium spp. Proc. Natl. Acad. Sci. USA 2021, 118, e2013338118. [CrossRef]

15. Chen, W.; Zhang, Y.; Zhang, Y.; Pi, Y.; Gu, T.; Song, L.; Wang, Y.; Ji, Q. CRISPR/Cas9-based genome editing in Pseudo-monas aeruginosa and cytidine deaminase-mediated base editing in Pseudomonas Species. iScience 2018, 6, 222-231. [CrossRef] [PubMed]

16. Tong, Y.; Whitford, C.M.; Robertsen, H.L.; Blin, K.; Jørgensen, T.S.; Klitgaard, A.K.; Gren, T.; Jiang, X.; Weber, T.; Lee, S.Y. Highly efficient DSB-free base editing for streptomycetes with CRISPR-BEST. Proc. Natl. Acad. Sci. USA 2019, 116, 20366-20375. [CrossRef] [PubMed]

17. Engler, C.; Youles, M.; Gruetzner, R.; Ehnert, T.M.; Werner, S.; Jones, J.D.G.; Patron, N.J.; Marillonnet, S. A Golden Gate modular cloning toolbox for plants. ACS Synth. Biol. 2014, 3, 839-843. [CrossRef] [PubMed]

18. Thuronyi, B.W.; Koblan, L.W.; Levy, J.M.; Yeh, W.H.; Zheng, C.; Newby, G.A.; Wilson, C.; Bhaumik, M.; Shubina-Oleinik, O.; Holt, J.R.; et al. Continuous evolution of base editors with expanded target compatibility and improved activity. Nat. Biotechnol. 2019, 37, 1070-1079. [CrossRef]

19. Zong, Y.; Song, Q.; Li, C.; Jin, S.; Zhang, D.; Wang, Y.; Qiu, J.-L.; Gao, C. Efficient C-to-T base editing in plants using a fusion of nCas9 and human APOBEC3A. Nat. Biotechnol. 2018, 36, 950-953. [CrossRef]

20. Richter, M.F.; Zhao, K.T.; Eton, E.; Lapinaite, A.; Newby, G.A.; Thuronyi, B.W.; Wilson, C.; Koblan, L.W.; Zeng, J.; Bauer, D.E.; et al Phage-assisted evolution of an adenine base editor with improved Cas domain compatibility and activity. Nat. Biotechnol. 2020, 38, 883-891. [CrossRef]

21. Yan, D.; Ren, B.; Liu, L.; Yan, F.; Li, S.; Wang, G.; Sun, W.; Zhou, X.; Zhou, H. High-efficiency and multiplex adenine base editing in plants using new TadA variants. Mol. Plant 2021, 14, 722-731. [CrossRef] [PubMed]

22. Zhang, Y.; Zhang, H.; Wang, Z.; Wu, Z.; Wang, Y.; Tang, N.; Xu, X.; Zhao, S.; Chen, W.; Ji, Q. Programmable adenine de-amination in bacteria using a Cas9-adenine-deaminase fusion. Chem. Sci. 2020, 11, 1657-1664. [CrossRef] [PubMed]

23. Zheng, K.; Wang, Y.; Li, N.; Jiang, F.-F.; Wu, C.-X.; Liu, F.; Chen, H.-C.; Liu, Z.-F. Highly efficient base editing in bacteria using a Cas9-cytidine deaminase fusion. Commun. Biol. 2018, 1, 32. [CrossRef]

24. Moore, S.J.; Lai, H.E.; Kelwick, R.J.R.; Chee, S.M.; Bell, D.J.; Polizzi, K.M.; Freemont, P.S. EcoFlex: A Multifunctional MoClo Kit for E. coli Synthetic Biology. ACS Synth. Biol. 2016, 5, 1059-1069. [CrossRef] [PubMed]

25. Lee, M.E.; DeLoache, W.C.; Cervantes, B.; Dueber, J.E. A highly characterized yeast toolkit for modular, multipart assembly. ACS Synth. Biol. 2015, 4, 975-986. [CrossRef]

26. Assaad, F.F.; Signer, E.R. Cauliflower mosaic virus P35S promoter activity in Escherichia coli. MGG Mol. Gen. Genet. 1990, 223, 517-520. [CrossRef]

27. Lewin, A.; Jacob, D.; Freytag, B.; Appel, B. Gene expression in bacteria directed by plant-specific regulatory sequences. Transgenic Res. 1998, 7, 403-411. [CrossRef]

28. Marshall, R.; Maxwell, C.S.; Collins, S.P.; Jacobsen, T.; Luo, M.L.; Begemann, M.B.; Gray, B.N.; January, E.; Singer, A.; He, Y.; et al Rapid and scalable characterization of CRISPR technologies using an E. coli cell-free transcription-translation system. Mol. Cell 2018, 69, 146-157.e3. [CrossRef]

29. Oh, Y.; Lee, B.; Kim, H.; Kim, S.G. A multiplex guide RNA expression system and its efficacy for plant genome engineering. Plant Methods 2020, 16, 37. [CrossRef]

30. Qi, L.S.; Larson, M.H.; Gilbert, L.A.; Doudna, J.A.; Weissman, J.S.; Arkin, A.P.; Lim, W.A. Repurposing CRISPR as an RNA-guided platform for sequence-specific control of gene expression. Cell 2013, 152, 1173-1183. [CrossRef] [PubMed]

31. Wang, Y.; Liu, Y.; Liu, J.; Guo, Y.; Fan, L.; Ni, X.; Zheng, X.; Wang, M.; Zheng, P.; Sun, J.; et al. MACBETH: Multiplex automated Corynebacterium glutamicum base editing method. Metab. Eng. 2018, 47, 200-210. [CrossRef] [PubMed]

32. Li, Q.; Seys, F.M.; Minton, N.P.; Yang, J.; Jiang, Y.; Jiang, W.; Yang, S. CRISPR-Cas9 D10A nickase-assisted base editing in the solvent producer Clostridium beijerinckii. Biotechnol. Bioeng. 2019, 116, 1475-1483. [CrossRef]

33. Arbab, M.; Shen, M.W.; Mok, B.; Wilson, C.; Matuszek, Ż.; Cassa, C.A.; Liu, D.R. Determinants of base editing outcomes from target library analysis and machine learning. Cell 2020, 182, 463-480.e30.

34. Lei, Z.; Meng, H.; Lv, Z.; Liu, M.; Zhao, H.; Wu, H.; Zhang, X.; Liu, L.; Zhuang, Y.; Yin, K.; et al. Detect-seq reveals out-ofprotospacer editing and target-strand editing by cytosine base editors. Nat. Methods 2021, 18, 643-651. [CrossRef] 
35. Katti, A.; Foronda, M.; Zimmerman, J.; Diaz, B.; Zafra, M.P.; Goswami, S.; Dow, L.E. GO: A functional reporter system to identify and enrich base editing activity. Nucleic Acids Res. 2020, 48, 2841-2852. [CrossRef]

36. Kim, D.-H.; Lee, J.; Suh, Y.; Lee, K. Necessity for validation of effectiveness of selected guide RNA in silico for application of CRISPR/Cas9. Mol. Biotechnol. 2021, 63, 140-149. [CrossRef]

37. Naim, F.; Shand, K.; Hayashi, S.; O’Brien, M.; McGree, J.; Johnson, A.A.T.; Dugdale, B.; Waterhouse, P.M. Are the current gRNA ranking prediction algorithms useful for genome editing in plants? PLoS ONE 2020, 15, e0227994. [CrossRef]

38. Hahn, F.; Mantegazza, O.; Greiner, A.; Hegemann, P.; Eisenhut, M.; Weber, A.P.M. An efficient visual screen for CRISPR/CAS9 activity in arabidopsis thaliana. Front. Plant Sci. 2017, 8, 39. [CrossRef]

39. Pramanik, D.; Shelake, R.M.; Park, J.; Kim, M.J.; Hwang, I.; Park, Y.; Kim, J.-Y. CRISPR/Cas9-mediated generation of pathogenresistant tomato against Tomato Yellow Leaf Curl Virus and powdery mildew. Int. J. Mol. Sci. 2021, 22, 1878. [CrossRef]

40. Thyme, S.B.; Akhmetova, L.; Montague, T.G.; Valen, E.; Schier, A.F. Internal guide RNA interactions interfere with Cas9-mediated cleavage. Nat. Commun. 2016, 7, 11750. [CrossRef]

41. Hao, W.; Cui, W.; Cheng, Z.; Han, L.; Suo, F.; Liu, Z.; Zhou, L.; Zhou, Z. Development of a base editor for protein evolution via in situ mutation in vivo. Nucleic Acids Res. 2021, 49, 9594-9605. [CrossRef]

42. Wang, Y.; Liu, Y.; Li, J.; Yang, Y.; Ni, X.; Cheng, H.; Huang, T.; Guo, Y.; Ma, H.; Zheng, P.; et al. Expanding targeting scope, editing window, and base transition capability of base editing in Corynebacterium glutamicum. Biotechnol. Bioeng. 2019, 116, 3016-3029. [CrossRef]

43. Jia, B.; Jeon, C.O. High-throughput recombinant protein expression in Escherichia coli: Current status and future perspectives. Open Biol. 2016, 6, 160196.

44. Shams, A.; Higgins, S.A.; Fellmann, C.; Laughlin, T.G.; Oakes, B.L.; Lew, R.; Kim, S.; Lukarska, M.; Arnold, M.; Staahl, B.T.; et al. Comprehensive deletion landscape of CRISPR-Cas9 identifies minimal RNA-guided DNA-binding modules. Nat. Commun. 2021, 12,5664 .

45. Lapinaite, A.; Knott, G.J.; Palumbo, C.M.; Lin-Shiao, E.; Richter, M.F.; Zhao, K.T.; Beal, P.A.; Liu, D.R.; Doudna, J.A. DNA capture by a CRISPR-Cas9-guided adenine base editor. Science 2020, 369, 566-571.

46. Cheng, T.L.; Li, S.; Yuan, B.; Wang, X.; Zhou, W.; Qiu, Z. Expanding C-T base editing toolkit with diversified cytidine de-aminases. Nat. Commun. 2019, 10, 3612. [CrossRef]

47. Kurt, I.C.; Zhou, R.; Iyer, S.; Garcia, S.P.; Miller, B.R.; Langner, L.M.; Grünewald, J.; Joung, J.K. CRISPR C-to-G base editors for inducing targeted DNA transversions in human cells. Nat. Biotechnol. 2021, 39, 41-46.

48. Salter, J.D.; Bennett, R.P.; Smith, H.C. The APOBEC protein family: United by structure, divergent in function. Trends Biochem. Sci. 2016, 41, 578-594. [CrossRef]

49. Gaudelli, N.M.; Komor, A.C.; Rees, H.A.; Packer, M.S.; Badran, A.H.; Bryson, D.I.; Liu, D.R. Programmable base editing of A $\bullet$ T to $\mathrm{G} \bullet \mathrm{C}$ in genomic DNA without DNA cleavage. Nature 2017, 551, 464-471.

50. Salter, J.D.; Smith, H.C. Modeling the embrace of a mutator: APOBEC Selection of nucleic acid ligands. Trends Biochem. Sci. 2018, $43,606-622$.

51. Jansing, J.; Schiermeyer, A.; Schillberg, S.; Fischer, R.; Bortesi, L. Genome editing in agriculture: Technical and practical considerations. Int. J. Mol. Sci. 2019, 20, 2888. [CrossRef]

52. Lee, M.H.; Lee, J.; Choi, S.A.; Kim, Y.-S.; Koo, O.; Choi, S.H.; Ahn, W.S.; Jie, E.Y.; Kim, S.W. Efficient genome editing using CRISPR-Cas9 RNP delivery into cabbage protoplasts via electro-transfection. Plant Biotechnol. Rep. 2020, 14, 695-702. [CrossRef]

53. Huang, X.; Wang, Y.; Xu, J.; Wang, N. Development of multiplex genome editing toolkits for citrus with high efficacy in biallelic and homozygous mutations. Plant Mol. Biol. 2020, 104, 297-307. [CrossRef] [PubMed]

54. Fan, Y.; Xin, S.; Dai, X.; Yang, X.; Huang, H.; Hua, Y. Efficient genome editing of rubber tree (Hevea brasiliensis) protoplasts using CRISPR/Cas9 ribonucleoproteins. Ind. Crops Prod. 2020, 146, 112146. [CrossRef]

55. Nicolia, A.; Andersson, M.; Hofvander, P.; Festa, G.; Cardi, T. Tomato protoplasts as cell target for ribonucleoprotein (RNP)mediated multiplexed genome editing. Plant Cell Tissue Organ Cult. 2021, 144, 463-467. [CrossRef]

56. Badhan, S.; Ball, A.S.; Mantri, N. First report of CRISPR/Cas9 mediated DNA-free editing of $4 C L$ and RVE7 genes in chickpea protoplasts. Int. J. Mol. Sci. 2021, 22, 396. [CrossRef]

57. Kaur, M.; Manchanda, P.; Kalia, A.; Ahmed, F.K.; Nepovimova, E.; Kuca, K.; Abd-Elsalam, K.A. Agroinfiltration mediated scalable transient gene expression in genome edited crop plants. Int. J. Mol. Sci. 2021, 22, 10882. [CrossRef]

58. Isaac, R.S.; Jiang, F.; Doudna, J.A.; Lim, W.A.; Narlikar, G.J.; Almeida, R. Nucleosome breathing and remodeling constrain CRISPR-Cas9 function. Elife 2016, 5, e13450. [CrossRef]

59. Weber, E.; Engler, C.; Gruetzner, R.; Werner, S.; Marillonnet, S. A modular cloning system for standardized assembly of multigene constructs. PLoS ONE 2011, 6, e16765. [CrossRef]

60. Livak, K.J.; Schmittgen, T.D. Analysis of relative gene expression data using real-time quantitative PCR and the $2^{-\Delta \Delta C T}$ method. Methods 2001, 25, 402-408. [CrossRef]

61. Kluesner, M.G.; Nedveck, D.A.; Lahr, W.S.; Garbe, J.R.; Abrahante, J.E.; Webber, B.R.; Moriarity, B.S. EditR: A method to quantify base editing from Sanger sequencing. CRISPR J. 2018, 1, 239-250. [CrossRef] [PubMed] 